Estudio y aplicación de diferentes metodologías de cálculo para el conocimiento gradual del proceso constructivo y comportamiento mecánico de una arquería de la Mezquita de Córdoba
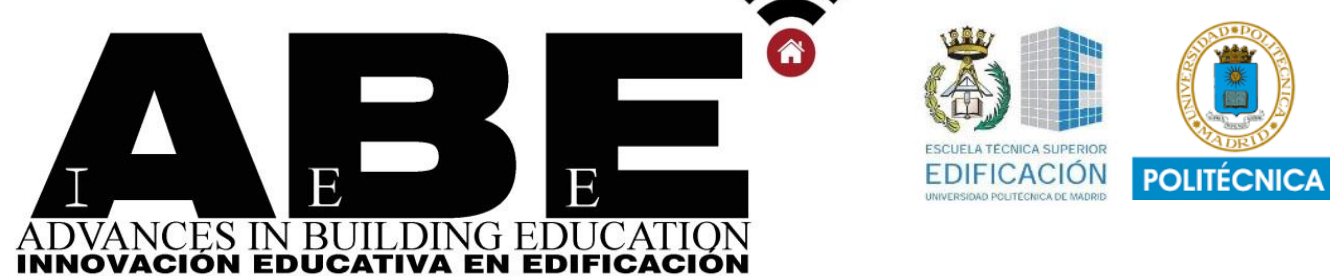

\title{
Estudio y aplicación de diferentes metodologías de cálculo para el conocimiento gradual del proceso constructivo y comportamiento mecánico de una arquería de la Mezquita de Córdoba
}

Study and application of different calculation methodologies for the gradual knowledge of the construction process and mechanical behavior of an arcade of the Mosque of Cordoba

\footnotetext{
María Escalada Marco-Gardoqui ${ }^{{ }^{*}}$

${ }^{1}$ MUCTEH - Máster Universitario en Construcción y Tecnología de Edificios Históricos.

Escuela Técnica Superior de Arquitectura. Universidad Politécnica de Madrid.

*Corresponding author email: mescaladamg@gmail.com
}

\section{TITULARES}

- Aprendizaje autónomo con software básico de programación matemática

- Planteamiento constructivo como fundamento básico del cálculo estructural

- Resolución de un problema con diferentes técnicas graduadas en complejidad

- Apoyo gráfico que facilita la compresión del cálculo complejo

\section{HIGHLIGHTS}

- Self-learning using basic mathematical programming software

- Constructive approach as a basis for structural calculation

- Solving a problem with different techniques increasing complexity

- Graphic support that facilitates the understanding of complex calculation 


\begin{abstract}
Estudio y aplicación de diferentes metodologías de cálculo para el conocimiento gradual del proceso constructivo y comportamiento mecánico de una arquería de la Mezquita de Córdoba María Escalada Marco-Gardoqui
\end{abstract}

\title{
RESUMEN
}

La mecánica de las estructuras de fábrica ha sido durante mucho tiempo de alguna manera abandonada en la enseñanza universitaria durante la licenciatura de arquitectura, en comparación con otros campos del conocimiento, como el hormigón armado, el acero y la madera. Una parte importante de nuestro patrimonio arquitectónico se erigió empleando estas técnicas ampliamente difundidas antaño, pero ahora olvidadas tanto en la práctica constructiva como en el marco teórico de la universidad, más centrado en tecnologías contemporáneas e innovadoras. Los avances del software computacional como son los modelos macro/micro de elementos finitos nos permiten realizar un análisis extremadamente complejo de estructuras de mampostería, pero paradójicamente, al mismo tiempo, se distancian de las limitaciones y la forma en que los antiguos concibieron y entendieron la arquitectura. Los maestros canteros fueron capaces de construir perfectamente sin la ayuda de tales pruebas precisas sobre los materiales. Por lo tanto, la mentalidad que hay que adoptar a la hora de abordar la enseñanza del comportamiento de las estructuras de mampostería es aquella en la que se estimule al alumnado a apropiarse del conocimiento no solo de la geometría, sino también y principalmente de las fases del proceso constructivo y de las técnicas acordes al período histórico. Solo una vez elaboradas ciertas hipótesis sobre ellas, se deberá comenzar con el cálculo gráfico-numérico. El objetivo del presente trabajo es exponer una metodología de enseñanza basada en programas de optimización y ejemplificada con el estudio de un caso concreto: el análisis de las arquerías de la Mezquita de Córdoba y de los posibles sistemas de contrarresto provisionales de empujes mediante tirantes y apeos, buscando la rentabilidad material de los mismos. Se plantea un recorrido progresivo, con un gradiente de conocimiento, a través de un estudio gráfico-numérico que engloba cuatro programas de diferente complejidad: Geogebra, Autocad + Excel, Maple y Sap2000, partiendo siempre de una hipótesis constructiva razonada. De esta manera, se resuelve el mismo problema de maneras diferentes para entender la estática gráfica desde varias perspectivas y ver cómo agilizar y "sistematizar" el cálculo de este tipo de estructuras mediante modelos elaborados con software de optimización matemática y programación simbólica, aprendiendo a la vez, cuáles son sus limitaciones y dificultades.

Palabras clave: Educación universitaria de posgrado, aprendizaje autónomo, proceso constructivo, arquerías Mezquita de Córdoba, estática gráfica, optimización matemática.

\section{ABSTRACT}

The mechanics of masonry structures have been for a long time pushed aside in university teaching during the bachelor degree of architecture in comparison with other fields of knowledge, such as reinforced concrete, steel and wood. An important part of our building heritage is raised using techniques once widely spread but now forgotten in the construction practice as well as in the theoretical university framework, more focused on contemporary and innovative technologies. Developments regarding computational software like FEM macro/micro modelling and discrete element methods allow us nowadays to carry out extremely complex analysis of accomplished masonry structures. Paradoxically, at the same time, this distances itself from the limitations and the way ancients conceived and understood architecture. Master bricklayers and stonemasons were able to build wonderfully without the help of such precise tests on the materials. Therefore, the mind-set when tackling the teaching of masonry structures should be that one where it is forced to the student body to take ownership of the knowledge of the geometry, constructive techniques and processes according to the precise historic period, keeping the tradition alive.

Advances in Building Education / Innovación Educativa en Edificación | ISSN: 2530-7940 |

http://polired.upm.es/index.php/abe

| Cod. 0021 | Septiembre - Diciembre 2017 | Vol. 1. № 3 | pp. 80/106 | 
Estudio y aplicación de diferentes metodologías de cálculo para el conocimiento gradual del proceso constructivo y comportamiento mecánico de una arquería de la Mezquita de Córdoba María Escalada Marco-Gardoqui

The purpose of this work is to present a teaching methodology mainly based on optimization programs for masonry structures. It is illustrated with the analysis of the construction process of the colonnaded portico with double arches of the Great Mosque of Cordoba. The requirement of hypothetical auxiliary resources, such as wood props for the fixation of the capitals and tightening structures will also be considered. This methodology should be understood as a progressive path with a knowledge gradient through a graphical-numerical study that involves the use of four programs: Geogebra, Autocad + Excel, Maple and Sap2000. In this way, the same problem of the thrust line falling within the boundaries of the archery is solved in various ways to understand graphic statics from different perspectives. Moreover, creating a personal optimization model sheet will be taught as a way of accelerating and "systematizing" the calculation. The study will be followed by more complex analysis using symbolic calculation programs. As a result of the learning process described, the limitation and difficulties of each way of approaching the stability problem of the archery will be understood. Not only that, but also students will take advantage of the freedom offered when proceeding to the limit analysis suited to their own interests, always having graphical support.

Key words: Postgraduate university education, self-learning, constructive process, arches Mosque of Cordoba, graphic statics, mathematical optimization

\section{INTRODUCCIÓN}

En el proceso constructivo de una fila de arquerías de la mezquita de Córdoba, el problema principal es mantener la estabilidad de un sistema que solo acaba de equilibrarse una vez que se ha completado todo su desarrollo y construido todos los arcos, hasta entrar en contacto con los muros perimetrales y sus contrafuertes. El objetivo del presente trabajo es el estudio de los sistemas de contrarresto provisionales de empujes mediante tirantes y apeos, buscando la eficiencia de los mismos desde el punto de vista de economía de material y de reutilización. Para ello, se ha elaborado un estudio gráfico-numérico que engloba los siguientes programas: Geogebra, Autocad+Excel, Maple y Sap2000, trazando así un hilo conductor del trabajo de menor a mayor complejidad para la resolución del mismo problema y poder entender mejor las limitaciones y capacidades de cada uno. [1]

La hipótesis que se maneja es la de un orden de ejecución por tramos, principalmente, para poder utilizar un apoyo común para las cimbras de cada uno de los niveles, en el primero, para un arco de herradura, y en el segundo, para un arco de medio punto. La misma estructura de puntales serviría para apoyar las dos cimbras de un vano, logrando así una simplificación de los medios auxiliares y una reducción del plazo de ejecución. Se ha planteado una posible estructura de madera de dos pisos (figuras 1 y 2), con una parte inferior arriostrada con diagonales. Debido a que el arco superior es más grueso que el inferior, los puntales suben a ambos lados del primer arco sin interferir en su cimbra. Exteriormente a los dos castilletes de madera, se dispondrían los tirantes provisionales necesarios para el contrarresto abrazando las pilastras. El desmontaje de los mismos debería ser independiente del de las cimbras, y como mínimo, se considera que deberían atirantarse dos vanos seguidos, el que se está construyendo y el anterior, sobre el que aún no se ha dispuesto el muro y la cubierta. Una medida de seguridad (habría que determinar el coste una vez estimadas las secciones) es la de mantener el atirantado de todos los vanos hasta contrarrestar los empujes con un extremo de muro o contrafuerte pertenecientes a los cerramientos de la mezquita, una vez completada la arquería. 
Estudio y aplicación de diferentes metodologías de cálculo para el conocimiento gradual del proceso constructivo y comportamiento mecánico de una arquería de la Mezquita de Córdoba María Escalada Marco-Gardoqui

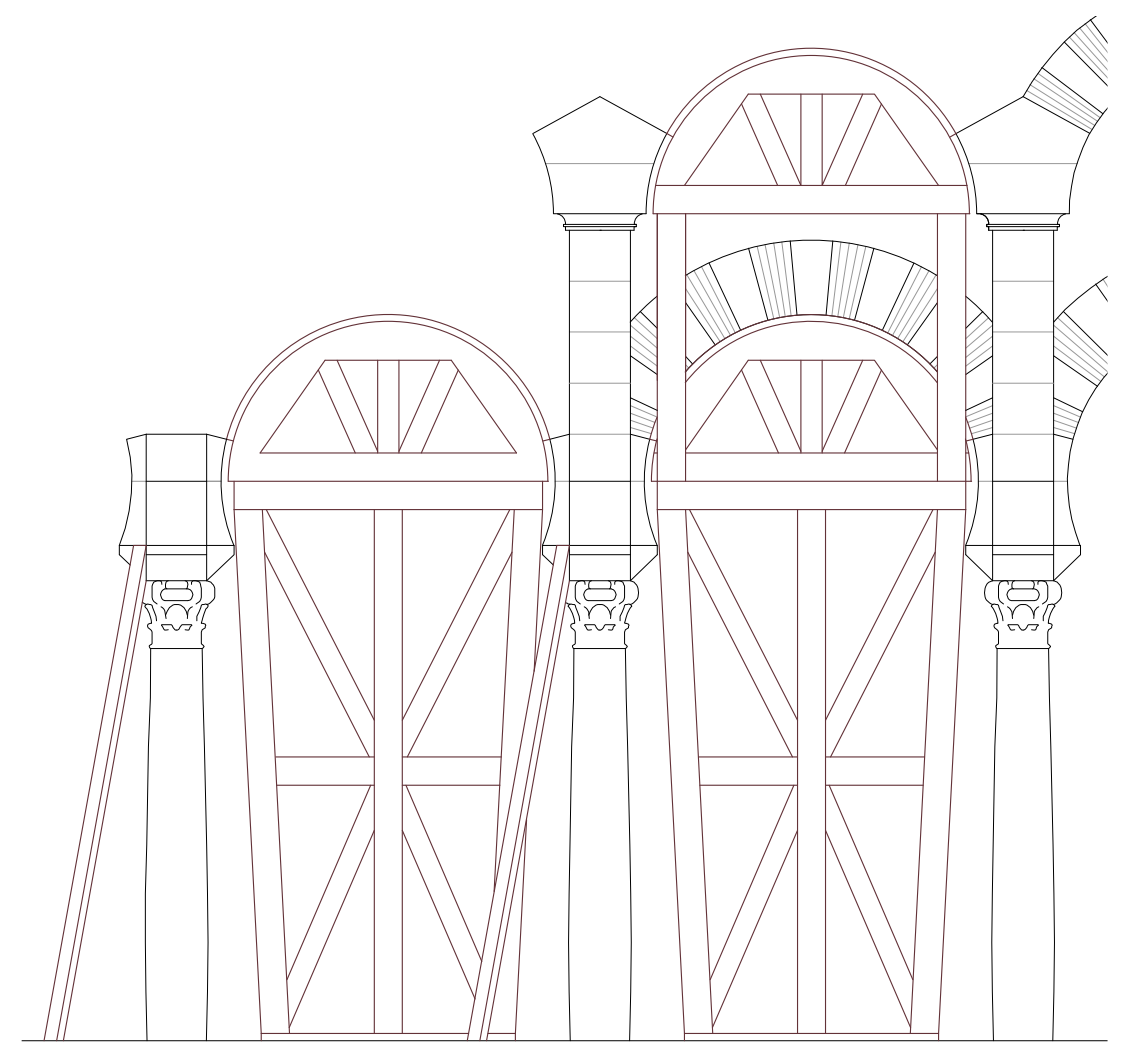

Fig. 1. Alzado con medios auxiliares de construcción propuestos. Elaboración propia.

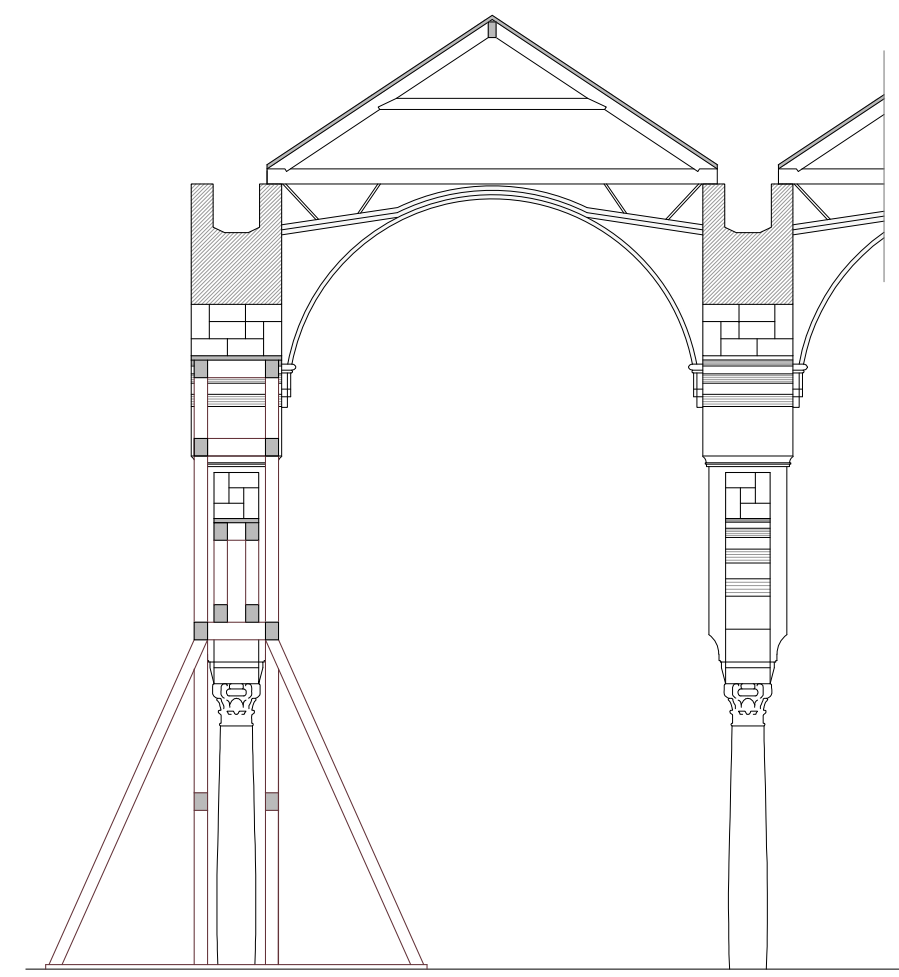

Fig. 2. Sección con medios auxiliares de construcción propuestos. Elaboración propia

Advances in Building Education / Innovación Educativa en Edificación | ISSN: 2530-7940 | http://polired.upm.es/index.php/abe

| Cod. 0021 | Septiembre - Diciembre 2017 | Vol. 1. № 3 | pp. 80/106 | 
Además del apuntalamiento, soporte de cimbras y otras plataformas, es posible que existiera un andamiaje paralelo para los propios constructores, probablemente móvil, con sus correspondientes sistemas de polipastos para la elevación de materiales. Se considera que la elevación del muro de ladrillo sobre el segundo nivel de arcos se realizaría de seguido e iría avanzando progresivamente una vez completado cada vano. De este modo, se podría ir componiendo la cubierta de madera apoyándose en dos arquerías, e ir protegiendo los muros recién construidos del sol y de la lluvia. Al mismo tiempo, la estructura de cubierta colaboraría en el atado entre dos arquerías paralelas y mejoraría su estabilidad en el sentido transversal.

A partir de este análisis inicial personal de la construcción de las arquerías, se enumeran una serie de posibles pasos a seguir, que ayudan a pensar dónde y cuándo podrán surgir problemas y necesidades de contrarresto. A la vez, esto ayuda a mejorar el planteamiento de la cimbra y de los propios tirantes y puntales, para que no entorpezcan la construcción de los arcos colindantes.

\section{Fases}

1. Apuntalamiento de la siguiente columna

2. Colocación del castillete de la cimbra inferior

3. Elevación de la pilastra sobre la columna

4. ¿Colocación del tirante? - Comprobar su necesidad en el cálculo

5. ¿Colocación de plataformas laterales de trabajo?

6. Construcción del arco de herradura

7. Levante del segundo piso del castillete y de la cimbra superior
8. Colocación de las piezas de arranque del arco de medio punto

9. ¿Apuntalamiento de la coronación de la pilastra y a continuación retirada del mismo apuntalamiento del tramo anterior? Valorar, - Comprobar su necesidad en el cálculo

10. Construcción del arco superior de medio punto

11. Apuntalamiento de la siguiente columna

12. Colocación del castillete de la cimbra inferior del siguiente vano

13. Elevación de la pilastra

14. ¿Colocación del tirante? - Comprobar su necesidad en el cálculo

15. ¿Colocación de plataformas laterales de trabajo?

16. Construcción del siguiente arco de herradura

17. Avance en la construcción del muro sobre el arco de medio punto del tramo anterior

18. Construcción de la cubierta sobre este muro levantado .... y así, sucesivamente.

Se ha intentado reflejar las dos fases descritas en los planos adjuntos a continuación (sin sistemas de contrarresto) y en el apartado de cálculo, incluyéndose las soluciones más adecuadas consideradas y otras hipótesis valoradas mediante croquis a mano.

\section{DISPOSITIVO EXPERIMENTAL}

\subsection{GEOGEBRA}

Para una primera toma de contacto con el funcionamiento de una arquería y un trabajo más intuitivo [2] y sencillo, se propone una modelización de la geometría mediante el 
programa Geogebra. Su facilidad de manejo permite abordar el problema planteado desde la experimentación [3] y la deducción de resultados en base a la modificación de los diferentes elementos (vectores, rayos polares $y$ geometría) que en él participan. A continuación, se muestra la mecánica seguida exclusivamente para el arco de herradura [4].

Se dibujan los polígonos de cada una de las dovelas (figura 3), y demás piezas, y se sitúan a continuación, su centro de gravedad y las líneas de acción perpendiculares a la línea de tierra. Los vectores del peso propio son valores invariables de acuerdo con el planteamiento de este ejercicio y su módulo se encuentra a una escala de $1 / 10(m)$, y poder tener una mejor visualización del gráfico. Se dibujan los vectores equipolentes del polígono de fuerzas en un ligar cualquiera del plano. Una vez colocados en vertical, se sitúa a su izquierda un punto focal móvil desde el que van a salir los rayos polares. Primero, se trazan las rectas que pasan por ese punto focal y por el inicio y fin de cada uno de los vectores. A continuación, se dibujan las resultantes inclinadas del sumatorio de fuerzas que actúan en el arco. En la línea de acción de la dovela D4, se coloca un "punto en objeto" que se denomina DES, para establecer la restricción de que este solo pueda desplazarse a lo largo de esa línea. Por él se traza una "recta paralela" al segundo de los rayos polares hasta que intersecte con la línea de acción de la dovela d5 y así sucesivamente, hasta lograr trazar la línea de empujes completa, que se intentará contener en el núcleo central de inercia. El criterio vendrá explicado en apartados posteriores.

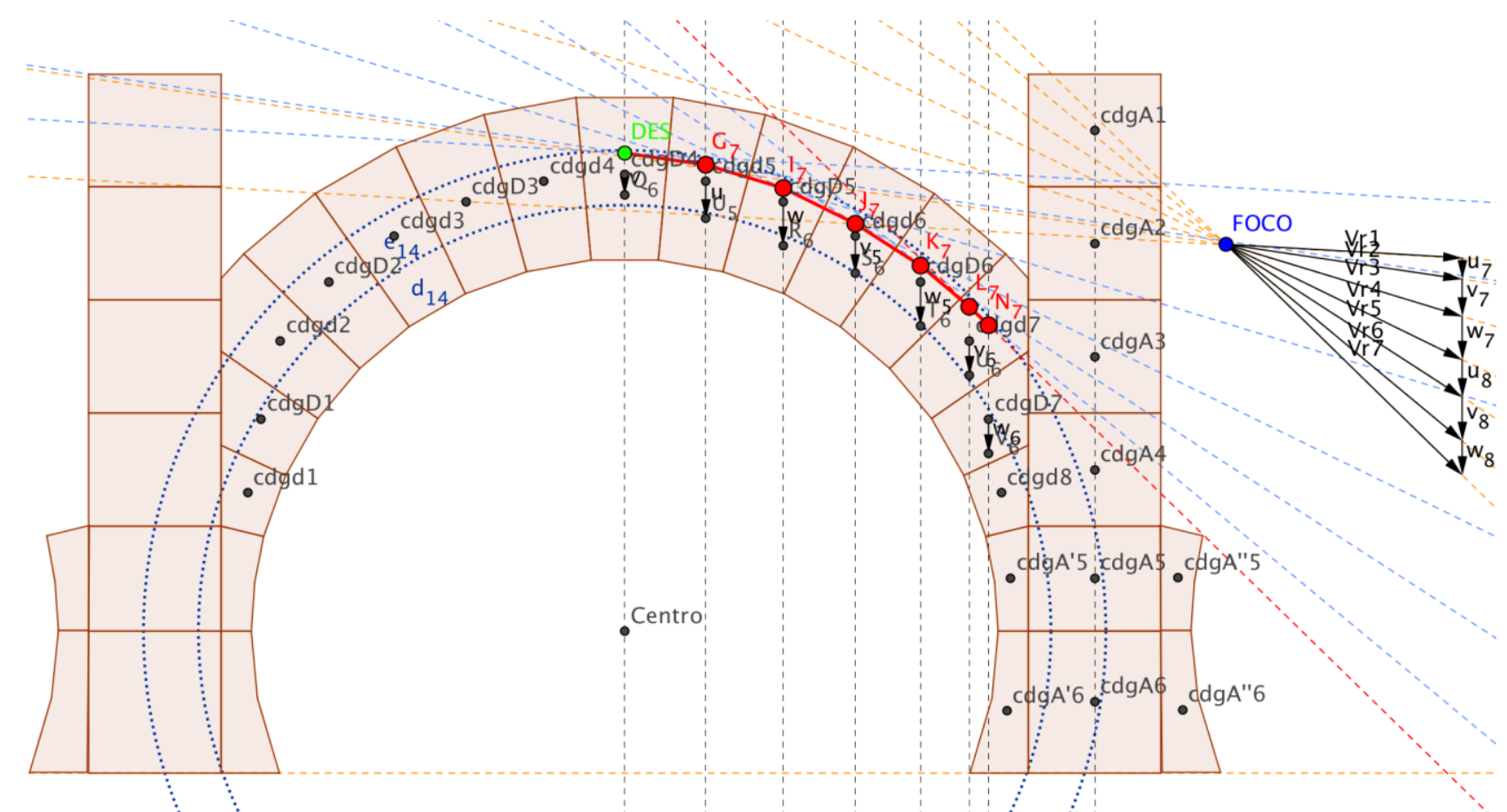

Fig. 3. Gráfica de Geogebra con el proceso de trazado de la línea de empujes. Elaboración propia.

Advances in Building Education / Innovación Educativa en Edificación | ISSN: 2530-7940 | http://polired.upm.es/index.php/abe

| Cod. 0021 | Septiembre - Diciembre 2017 | Vol. 1. № 3 | pp. 80/106 | 
Estudio y aplicación de diferentes metodologías de cálculo para el conocimiento gradual del proceso constructivo y comportamiento mecánico de una arquería de la Mezquita de Córdoba María Escalada Marco-Gardoqui

\subsection{EXCEL + AUTOCAD}

Partiendo de las conclusiones que se han podido extraer del modelo de Geogebra sobre la necesidad o no de sistemas de contrarresto de empujes para los arcos, se busca afinar en el trazado de la trayectoria sin renunciar a esa ventaja del software de encontrar las infinitas soluciones posibles, variando simplemente dos parámetros: el punto de paso inicial de la línea de empujes por la clave y la posición del foco que determina los ángulos de las fuerzas.

Por ello, se ha planteado un modelo de trabajo personal en Excel pensado para implementar la herramienta SOLVER [5], ofreciéndose esta como una alternativa a los programas especializados de optimización, como puede ser Maple, que requieren del aprendizaje de un lenguaje específico. Los pasos seguidos se especifican a continuación y su descripción permite comprender el contenido organizado de las tablas de resultados que se presentan en los apartados siguientes. Se tomará como modelo a explicar el caso del arco de herradura.

\subsubsection{Trazado en Autocad de una línea de empujes cualquiera}

A lo largo del trabajo pueden distinguir 3 apartados diferenciados: base, mínimo y máximo. Este punto 1 se refiere a la línea de empujes cualquiera [6] mostrada en la sección de "base" (figuras 4 y 5). En la línea de acción del peso propio de la clave, se escoge un punto cualquiera como origen del trazado. Al suponer la simetría total de la estructura de arcos, tanto geométrica como de acciones supuestas, se piensa llevar a cabo el estudio exclusivamente de la mitad del de herradura.
Dándole un ángulo aleatorio a la fuerza que sale de ese nudo, se realiza el esquema de fuerzas actuantes en ese punto D4, de tal forma que se obtenga el primer dibujo mostrado en la página siguiente.

Cuando esa resultante inclinada intersecta con la línea de acción vertical de la dovela siguiente $\mathrm{d} 5$, se hace el equilibrio en el nudo, incluyendo el nuevo peso propio y obteniéndose, por tanto, una resultante de mayor pendiente. Se repite este proceso hasta llegar al riñón del arco, que se corresponde con la dovela d7.

Es importante entender que, de acuerdo con el planteamiento de este ejercicio, no importa si la línea de empujes primera que se traza se sale de la geometría del arco. Lo que interesa son las formulaciones matemáticas de las ecuaciones de:

1. Las fuerzas inclinadas $F n y$ fn (nomenclatura que varía según se trate de una dovela de piedra o de ladrillo)

2. Las rectas verticales que pasan por los centros de gravedad de cada dovela, es decir, las líneas de acción de los pesos propios. 3. Los puntos de intersección entre ambas, cuya unión dará lugar al trazado de la línea de empujes.

\subsubsection{Obtención de las ecuaciones base}

\section{PASO 1}

Ecuación de la recta F4 (figura 6). Se busca la formulación paramétrica de la recta F4 (a partir de la continua), que pasa por un punto $A$ $(0,1.988)$ y sirve de origen para el trazado de la línea de empujes (en este caso, límite superior del núcleo central de inercia). 
Estudio y aplicación de diferentes metodologías de cálculo para el conocimiento gradual del proceso constructivo y comportamiento mecánico de una arquería de la Mezquita de Córdoba María Escalada Marco-Gardoqui

PASO 1

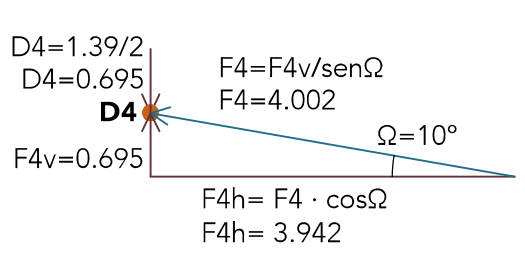

NOTA: El nudo no está en equilibrio, se movería hacia la izquierda a falta de una fuerza horizontal de igual módulo y sentido contrario que Fh. Si se dibujara el polígono de fuerzas, habría que representarla para cerrarlo.

PASO 2

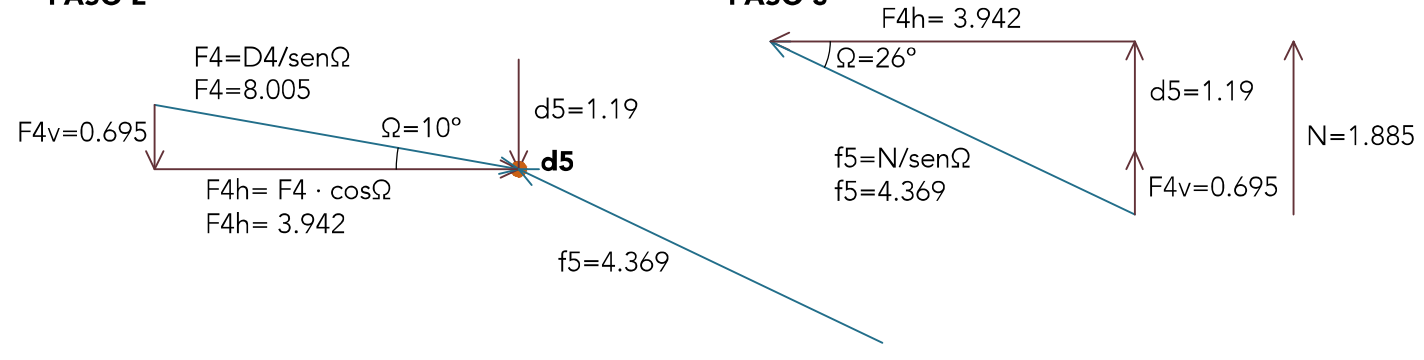

PASO 4

PASO 5

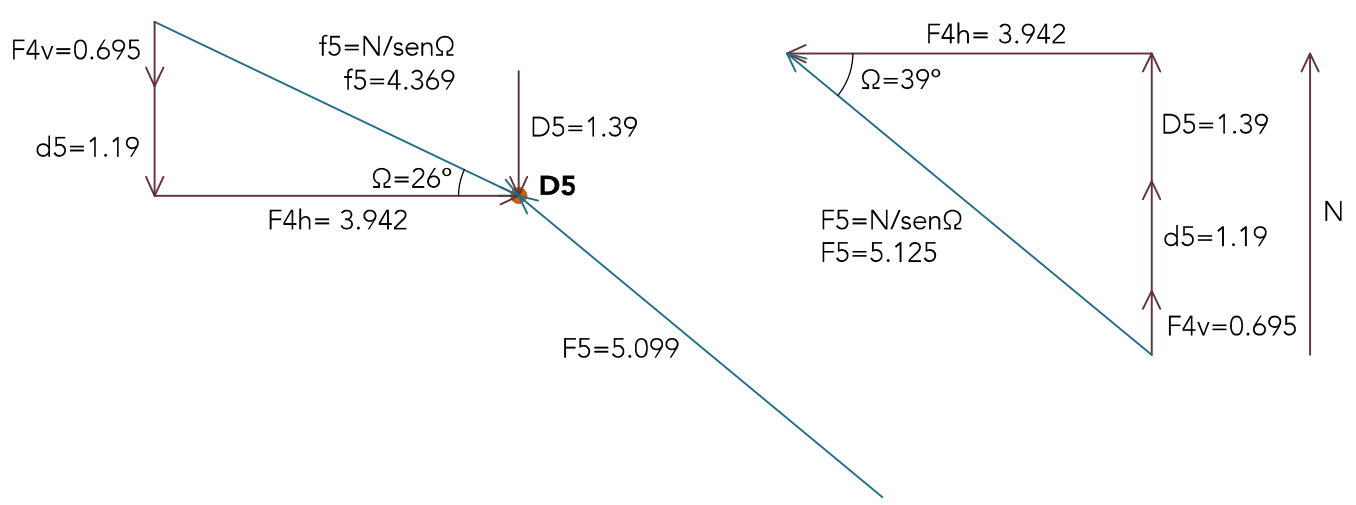

\section{PASO 6}

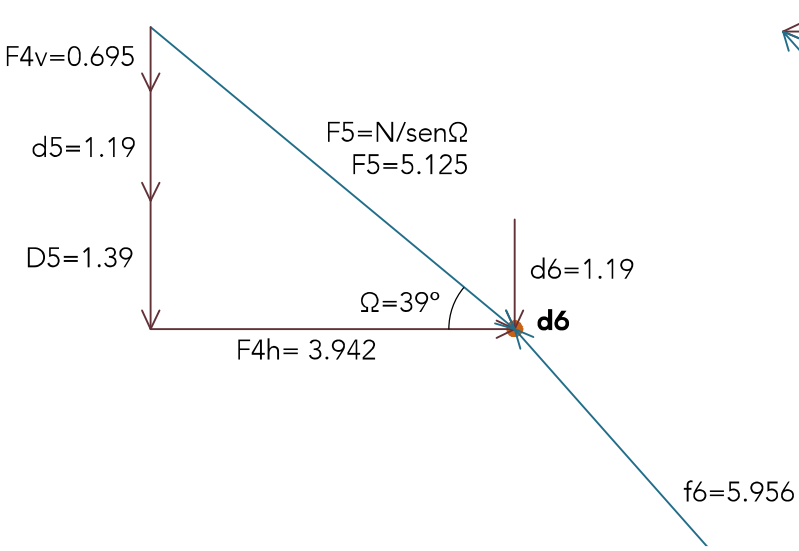

PASO 7

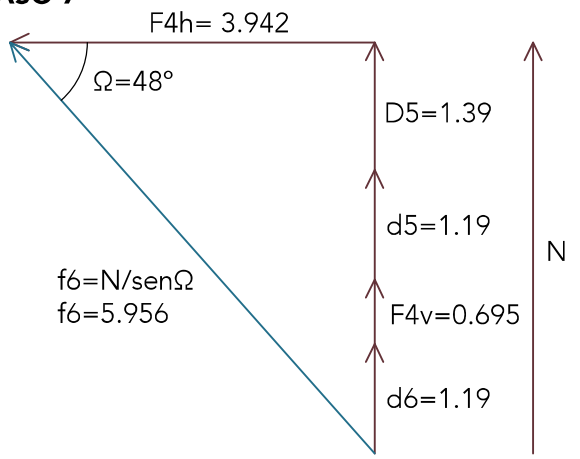

Fig. 4. Equilibrios en los nudos para línea de empujes de partida (I)

Advances in Building Education / Innovación Educativa en Edificación | ISSN: 2530-7940 | http://polired.upm.es/index.php/abe

| Cod. 0021 | Septiembre - Diciembre 2017 | Vol. 1. № 3 | pp. 80/106 | 
Estudio y aplicación de diferentes metodologías de cálculo para el conocimiento gradual del proceso constructivo y comportamiento mecánico de una arquería de la Mezquita de Córdoba María Escalada Marco-Gardoqui

PASO 10

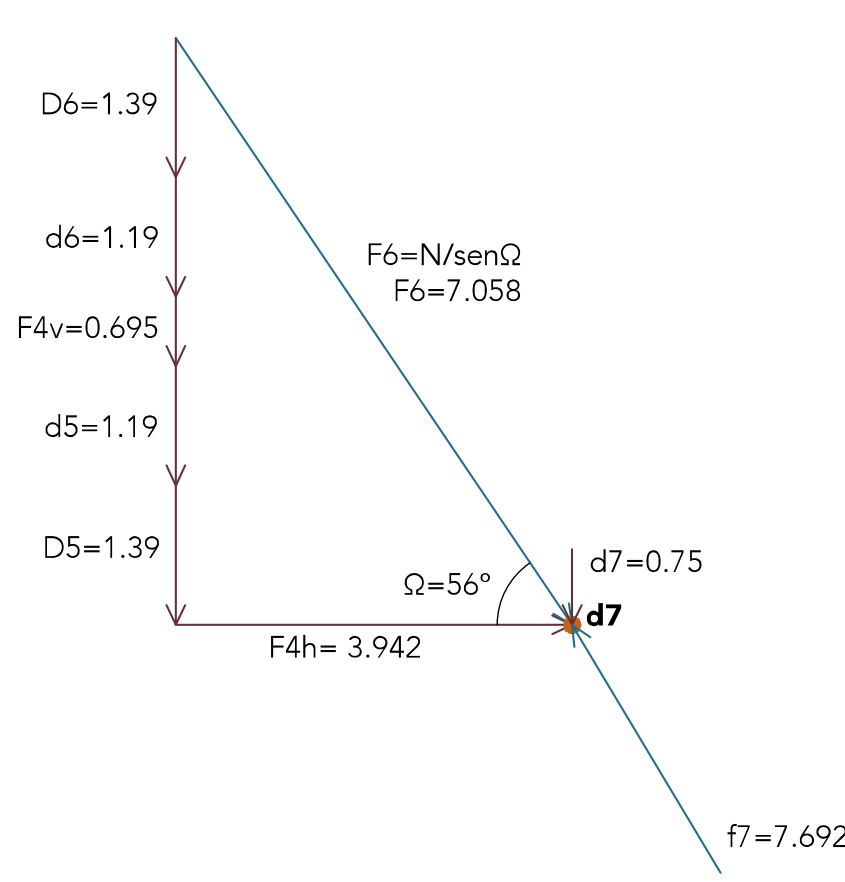

PASO 11

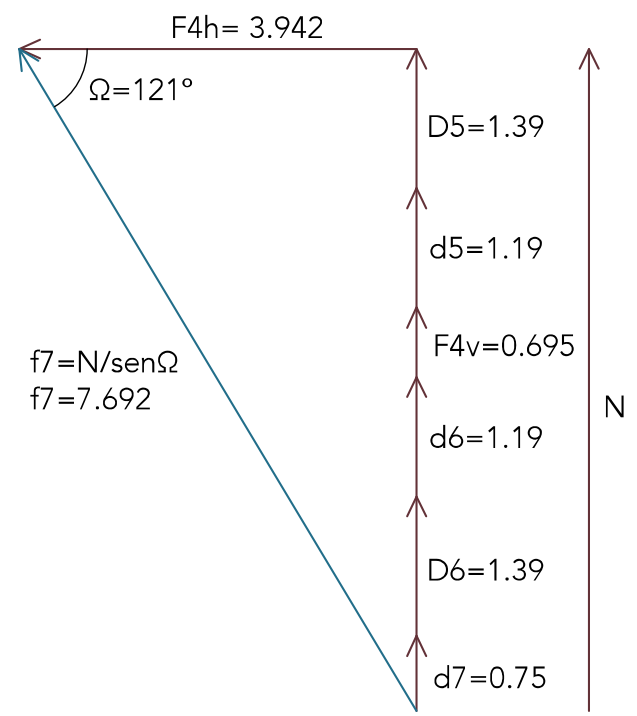

PASO 8

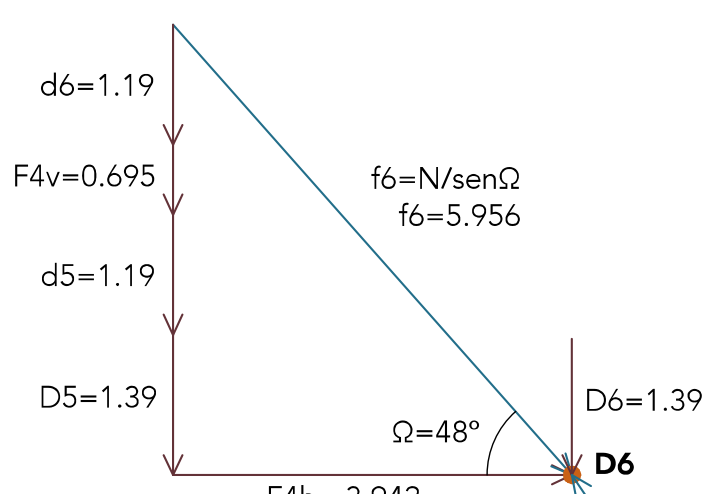

$F 4 h=3.942$
D6

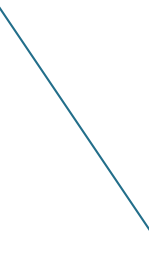

$\mathrm{F} 6=7.058$
PASO 9

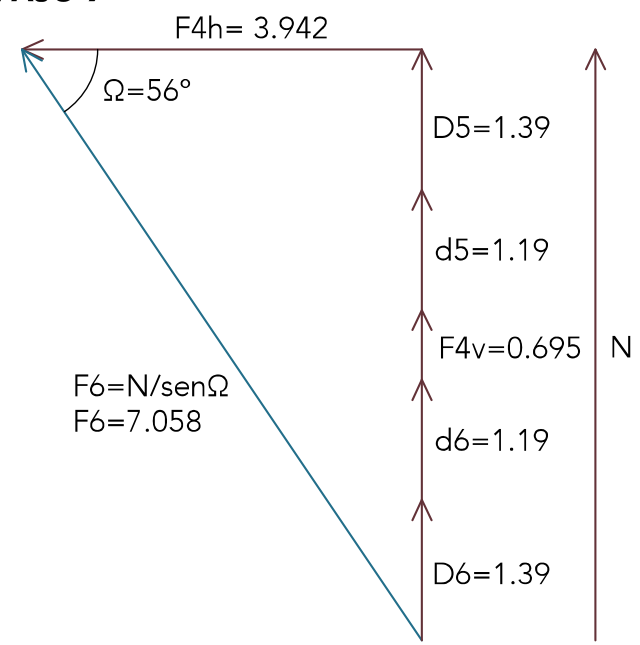

Fig. 5. Equilibrios en los nudos para línea de empujes de partida (II)

Advances in Building Education / Innovación Educativa en Edificación | ISSN: 2530-7940 | http://polired.upm.es/index.php/abe

| Cod. 0021 | Septiembre - Diciembre 2017 | Vol. 1. № 3 | pp. 80/106 | 
Estudio y aplicación de diferentes metodologías de cálculo para el conocimiento gradual del proceso constructivo y comportamiento mecánico de una arquería de la Mezquita de Córdoba María Escalada Marco-Gardoqui

Ecuación de la recta F4

$$
\begin{aligned}
& \frac{x-x 1}{v x}=\frac{y-y 1}{v y} \\
& \frac{x-0}{-3.942}=\frac{y-1.988}{0.695}
\end{aligned}
$$

$$
\begin{gathered}
0.695 x=-3.942(y-1.988) \\
0.695 x=-3.942 y+7.837 \\
0.695 x+3.942 y-7.837=0 \\
(A x+B y+C=0) \text { De donde: }
\end{gathered}
$$

$A=0.695 ; B=3.942 ; C=-7.837$

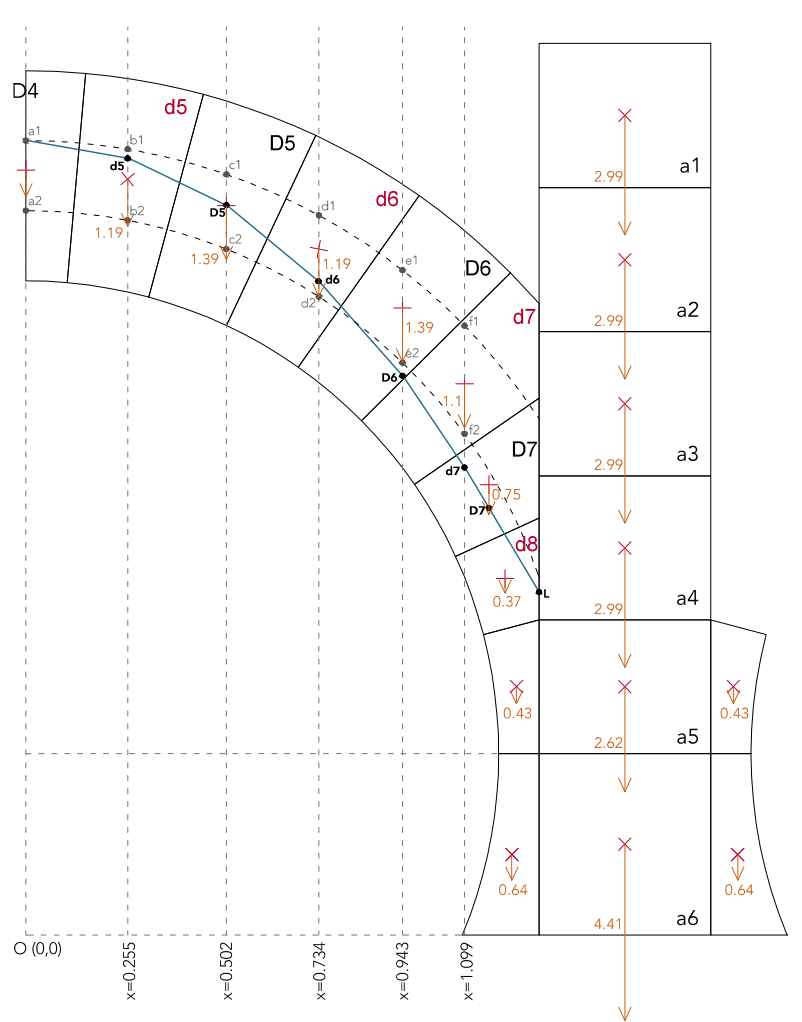

Fig. 6 Trazado de una línea de empujes cualquiera

De donde:

$$
A=1 ; B=0 ; C=-0.255
$$

\section{PASO 3}

Punto de intersección entre recta F4 y línea de acción d5. Se trata de resolver un sistema de dos ecuaciones con dos incógnitas:

\section{PASO 2}

Ecuación de la recta c.d.g. d5 (línea de acción). Se trata de una recta vertical que pasa por el punto $P(0,0.255)$, por lo que:

$$
\begin{aligned}
& \text { Ec1: } 0.695 x+3.942 y-7.837=0 \\
& \text { Ec2: } x-0.255=0 \\
& \text { intersección: } \\
& P(0.255,1.943)
\end{aligned}
$$$$
x=0.255
$$$$
x-0.255=0
$$$$
(A x+B y+C=0)
$$

Punto intersección: 
Este proceso habría que repetirlo dovela por dovela hasta llegar a trazar la línea de empujes completa para poder continuar con el problema. Tal y como se podrá observar más adelante, con conocimientos matemáticos básicos a partir de sencillos cortes de rectas en el plano se da solución a un problema gráfico bastante más complejo.

\subsubsection{Construcción del modelo de optimización en Excel}

Una vez obtenida la formulación matemática de las rectas y puntos de intersección, hay que buscar una forma de establecer las relaciones entre ellas, de determinar cuáles son los parámetros fijos o condicionantes, cuáles son las variables de decisión que nos permiten obtener infinitos resultados y, por último, cuál es la función objetivo, ese valor que se quiere optimizar (maximizar o minimizar).

Por un lado, se crea una tabla de tres grandes columnas en la que se definen las condiciones geométricas (figura 7). La central se reserva para las coordenadas $\mathrm{X}$ e $\mathrm{Y}$ de cada punto, fruto de la intersección entre cada fuerza inclinada y la línea de acción siguiente. A ambos valores se les fijan una serie de condicionantes (límites) de desplazamiento, es decir, se delimita un rango de soluciones posible que permita al programa obtener exclusivamente las infinitas líneas de empujes dentro del núcleo central de inercia. En $\mathrm{X}$, solo se permite un movimiento a lo largo de la línea de acción, de ahí que la abscisa de los puntos de intersección pueda tener un único valor. En $\mathrm{Y}$, sin embargo, el límite superior y el inferior son distintos puesto que esa cota de altura puede variar. ¿Cuánto? Siempre que entre dentro del tercio central. Estos valores máximos y mínimos se han extraído de la figura $3 b$ y reciben el nombre de n1y n2.

Este problema podría desarrollarse de la misma manera acotándolo a la anchura total del arco para trabajar con la teoría del análisis límite.

\begin{tabular}{|c|c|c|c|c|c|}
\hline \multicolumn{6}{|c|}{ Condiciones geométricas } \\
\hline \multicolumn{2}{|c|}{ Límites de coord. $x$} & \multicolumn{2}{|c|}{ P inicial - D4 } & \multicolumn{2}{|c|}{ Límites de coord. Y } \\
\hline$<$ & $>$ & $\mathbf{x}$ & y & $<$ & $>$ \\
\hline 0 & 0 & 0 & 1,813 & 1,988 & 1,813 \\
\hline & & \multicolumn{2}{|c|}{ d5 } & \multirow[t]{2}{*}{ (a1) } & \multirow[t]{2}{*}{ (a2) } \\
\hline & & $x$ & y & & \\
\hline \multirow[t]{3}{*}{0,255} & 0,255 & 0,255 & 1,768 & 1,967 & 1,789 \\
\hline & & \multicolumn{2}{|c|}{ D5 } & \multirow[t]{2}{*}{ (b1) } & \multirow[t]{2}{*}{ (b2) } \\
\hline & & $\mathbf{x}$ & y & & \\
\hline \multirow[t]{3}{*}{0,502} & 0,502 & 0,502 & 1,650 & 1,903 & 1,717 \\
\hline & & \multicolumn{2}{|c|}{ d6 } & \multirow[t]{2}{*}{ (c1) } & \multirow[t]{2}{*}{ (c2) } \\
\hline & & $\mathbf{x}$ & y & & \\
\hline \multirow[t]{3}{*}{0,734} & 0,734 & 0,734 & 1,457 & 1,801 & 1,598 \\
\hline & & \multicolumn{2}{|c|}{ D6 } & \multirow[t]{2}{*}{ (d1) } & \multirow[t]{2}{*}{ (d2) } \\
\hline & & $\mathbf{x}$ & $\mathbf{y}$ & & \\
\hline \multirow[t]{3}{*}{0,943} & 0,9432 & 0,943 & 1,220 & 1,664 & 1,432 \\
\hline & & \multicolumn{2}{|c|}{ d7 } & \multirow[t]{2}{*}{ (e1) } & \multirow[t]{2}{*}{ (e2) } \\
\hline & & $\mathbf{x}$ & $\mathbf{y}$ & & \\
\hline 1,0985 & 1,0985 & 1,099 & 0,989 & 1,525 & 1,255 \\
\hline
\end{tabular}

Fig. 7. Equilibrios en los nudos para línea de empuje mínimo (ya optimizado) (I) 
Estudio y aplicación de diferentes metodologías de cálculo para el conocimiento gradual del proceso constructivo y comportamiento mecánico de una arquería de la Mezquita de Córdoba María Escalada Marco-Gardoqui

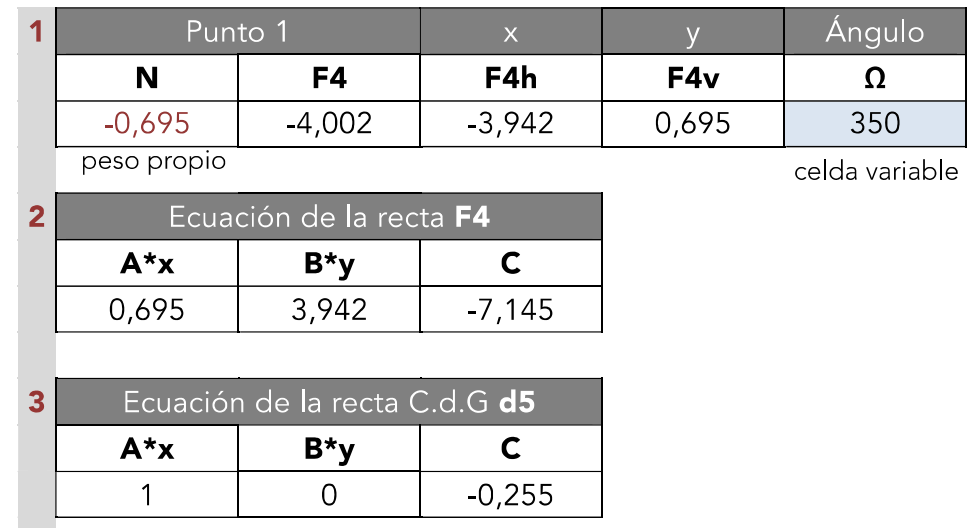

\begin{tabular}{|c|c|}
\hline \multicolumn{2}{|c|}{ Punto intersec. d5 } \\
\hline $\mathbf{x}$ & $\mathbf{y}$ \\
\hline 0,255 & 1,768 \\
\hline
\end{tabular}

\begin{tabular}{|c|c|c|c|c|}
\hline \multicolumn{2}{|c|}{ Punto 6 } & x & $y$ & Ángulo \\
\hline Peso & $\mathbf{f 7}$ & $\mathbf{f 7 h}$ & $\mathbf{f 7 v}$ & $\mathbf{\Omega}$ \\
\hline$-0,75$ & $-7,692$ & $-3,942$ & 6,605 & 300,83 \\
\hline \multicolumn{5}{|c|}{ celda objetivo } \\
\hline
\end{tabular}

Fig. 8. Equilibrios en los nudos para línea de empuje mínimo (ya optimizado) (II)

Se proponen las siguientes relaciones entre las ecuaciones matemáticas para cada una de las subdivisiones de la tabla que tienen por nombre 1, 2, 3, 4 en color granate. Para la dovela D4:

\section{INICIO:}

Descomposición de la resultante

Celda de $\mathrm{N}=$ Peso propio de la dovela $\mathrm{D} 4$, con signo negativo (Valor invariable)

Celda de F4 = F4v/ Seno (Radianes) $(\Omega)$

Celda de F4h $=$ F4 $\cdot$ Cos (Radianes) $(\Omega)$

Celda de F4v $=-\mathrm{N}$

Ángulo $=350^{\circ}$

\section{PASO 1:}

Ecuación de la recta F4

$A($ valor que multiplica a la $\mathrm{x})=\mathrm{F} 4 \mathrm{v}$
$B($ valor que multiplica a la $y)=-F 4 h$

$C$ (término independiente) $=-($ Coordenada $x$ del P. inicial D4) $\cdot F 4 v+($ Coordenada y del P. inicial D4) $\cdot$ F4h

\section{PASO 2:}

Ecuación de la recta c.d.g. d5 (línea de acción) (Valores invariables)
$A($ valor que multiplica a la $x)=1$
$B$ (valor que multiplica a la $y)=0$
C (término independiente) $=-0.255$

\section{PASO 3:}

Punto de intersección d5 entre recta F4 y línea de acción d5 $x=0.255$ (Valor del límite de coordenadas en $x$, que es invariable) 
Estudio y aplicación de diferentes metodologías de cálculo para el conocimiento gradual del proceso constructivo y comportamiento mecánico de una arquería de la Mezquita de Córdoba María Escalada Marco-Gardoqui

$y=-A($ ec. recta $F 4) \cdot x($ punto intersección d5)

- C (ec. recta F4) / B (ec. recta F4)

Una vez obtenidos todos los datos de los puntos intersección, estos se colocan en su posición correspondiente de la columna central de la tabla Condiciones Geométricas igualando celdas.

\subsubsection{Solver}

Introducidos los datos, el problema se resuelve ejecutando el comando Solver. Lo que se quiere conocer es el dato del mínimo y máximo empuje para determinar si es posible contenerlo dentro de la geometría del machón lateral y no tener la necesidad de disponer de un contrarresto provisional durante el proceso constructivo. Por ello, se establece como celda objetivo la f7h, esto es, la componente del empuje horizontal cuyo valor se piensa optimizar hacia máximos o mínimos. Las celdas variables son dos, tal y como se ha comentado ya con anterioridad y asemejándose al funcionamiento de la propuesta en Geogebra: el ángulo $\Omega$ inicial, que se había fijado en 350 o, y la coordenada en $Y$ del punto inicial D4. Serán los únicos valores que se podrán modificar en el punto de partida a la hora de optimizar el problema matemático.

El siguiente paso a seguir es la definición de las restricciones a las que están sujetas todos los puntos intersección, es decir, que no se salgan nunca del núcleo central de inercia. Para ello, se agregan condiciones exigidas para cada coordenada en $\mathrm{X}$ e $\mathrm{Y}$ de los puntos de la columna central de la tabla base de Condiciones Geométricas. Se señala la referencia de celda, se especifica el signo de la inecuación $<=0>=$, y a continuación la restricción, que son los límites inferior e inferior de la intersección de cada línea de acción con los arcos de circunferencia del núcleo central de inercia. Por ello, habrá para cada punto, 4 condiciones que restrinjan su movimiento.

Solo con todo esto listo, es posible mandar ejecutar la opción "Resolver".

\subsection{MAPLE}

Una aportación notable del modelo en Maple frente al trabajo previo realizado consiste en la introducción del coeficiente de rozamiento [7] necesario para la comprobación de la estabilidad de la arquería de fábrica. A la hora de obtener los resultados, se verificará que aquellos que demanda la estructura para permanecer en equilibrio según el programa, entran dentro del margen que se puede esperar encontrar en un sistema de este tipo (siempre teniendo en cuenta que los arcos siguen en pie hoy en día, por lo que cualquier resultado teórico que contradiga esta evidencia habrá de ser revisado).

Ante la imposibilidad de realizar ensayos precisos sobre los materiales de las dovelas y demás piezas que configuran la estructura objeto de análisis, se toma un rango de datos aceptable obtenido de la bibliografía clásica de referencia sobre el tema. Citando a Huertas, "Una última propiedad fundamental de las piedras es su elevado coeficiente de rozamiento. Esta característica, como se verá más adelante, es fundamental para dar cohesión a las fábricas. Este dato no se encuentra fácilmente en los modernos manuales de resistencia de materiales. Para los puentes de fábrica se suele tomar en casi todos los manuales $\mu=0,5(\Phi=27)$, valor que incluye un cierto coeficiente de seguridad". 
Estudio y aplicación de diferentes metodologías de cálculo para el conocimiento gradual del proceso constructivo y comportamiento mecánico de una arquería de la Mezquita de Córdoba María Escalada Marco-Gardoqui

Los valores que se pueden apreciar en la Tabla 1 de Coeficientes y ángulo de rozamiento entre las piedras, van a ser tomados como límites superiores a respetar a la hora de variar el parámetro en Maple [8].

La restricción a definir, por tanto, será la siguiente:

$$
\text { rstRoz: =rozamiento }(\text { miModelo, } \mathrm{Pi} / \mathrm{x})
$$

donde $\mathrm{Pi} / \mathrm{x}$ tendrá que tomar un valor siempre inferior a $39^{\circ}$. Una vez acotado dicho máximo, solo queda comenzar a ejecutar el algoritmo de programación lineal que resuelve el problema para la obtención del mínimo esfuerzo necesario (función objetivo) a introducir en la estructura para lograr la estabilidad del conjunto, de acuerdo con el método Simplex del BBMI.
En el apartado de la introducción se definió de acuerdo con un criterio de construcción previo al cálculo, la necesidad de apear los pilares. En el caso de la mezquita, debido a los entrantes cuadrangulares en las cuatro esquinas de los en los cimacios, se ha considerado acertado suponer que los puntales podrían apoyar en dichos rincones. Ahora bien, se trata, como bien se acaba de describir, de una decisión no fundamentada en resultados de cálculo y no abordada en los estudios parciales de estática gráfica llevados a cabo con Excel, por lo que se cree que es un buen momento para valorar cómo varía el comportamiento global de la estructura en caso de prescindir de ellos [8].

\begin{tabular}{cccc} 
Fuente & Piedra & $\Phi_{\circ}$ & $\mu(=\tan \Phi)$ \\
\hline Rondelet & piedra caliza grano fino & 30 & 0.58 \\
\hline Boistard & piedra caliza sup. picada & 38 & 0.78 \\
\hline Regnier & madera sobre piedra & 30 & 0.58 \\
\hline Perronet & piedra (sin especificar) & 39 & 0.81 \\
\hline Rennie & granito & 33 & 0.65 \\
\hline
\end{tabular}

Tabla 1. Coeficientes y ángulo de rozamiento entre las piedras

\section{CASO ESTUDIO A:}

Con cimacios apeados, calcular el empuje mínimo

\section{Caso A1:}

El método plástico establece, a través de sus teoremas (cinemático y estático) que una línea adecuada de empujes que equilibre la carga (que en este caso es exclusivamente el peso propio de los elementos) y esté contenida dentro de la fábrica garantiza la estabilidad de la arquería para dichas acciones actuantes. Por ello, bajo este criterio de coeficientes de seguridad geométricos de 1 en soportes y arcos, se intenta conocer cuál es el ángulo de 
Estudio y aplicación de diferentes metodologías de cálculo para el conocimiento gradual del proceso constructivo y comportamiento mecánico de una arquería de la Mezquita de Córdoba María Escalada Marco-Gardoqui

rozamiento mínimo necesario para lograr la estabilidad del conjunto. Se parte del límite inferior de $30 \mathrm{o}$, es decir $\mathrm{Pi} / 6$ y viendo que existe una solución factible al problema, se intenta optimizar dicho ángulo hacia mínimos, obteniendo un valor límite de $\mathrm{Pi} / 9.95=18.09^{\circ}$ $=0.315 \mathrm{rad}$. Se trata de un valor muy bajo que casi con total seguridad pueden proporcionarlo los propios lechos de la estructura con un acabado superficial con gradina.

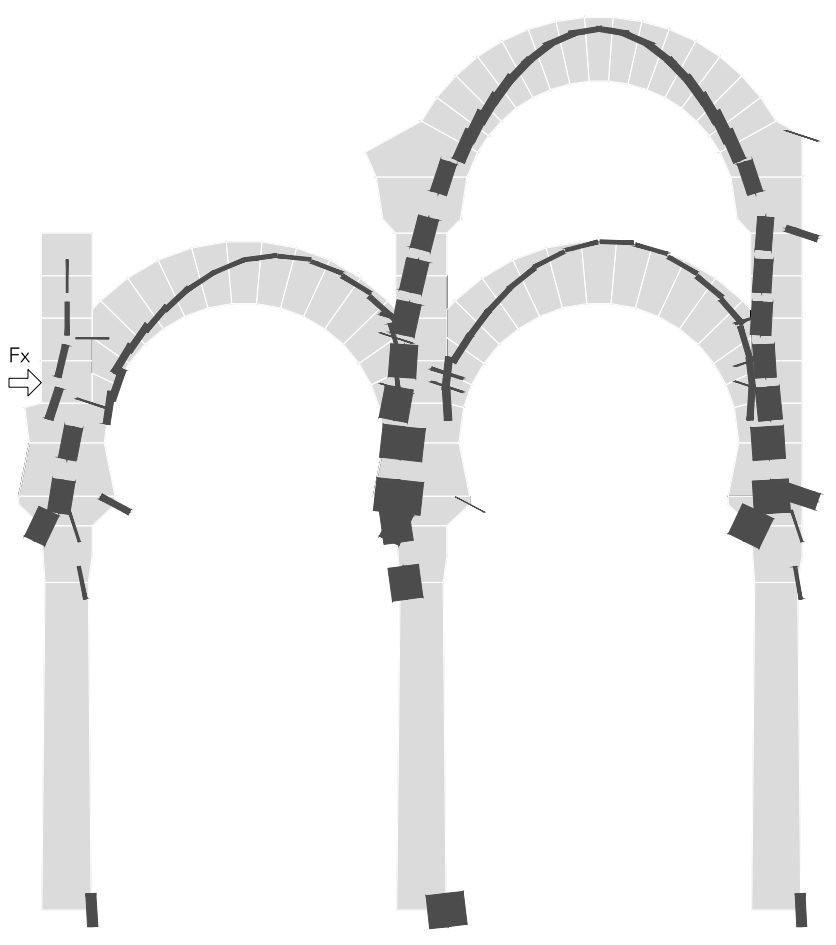

Fig. 9. CASO A1. Trayectoria de cargas para $C S G=1$, Ángulo de rozamiento: Pi/9.95 $(F x=0)$

Nota 1: Si se observan comparativamente las líneas de empujes fruto de la variación del coeficiente de rozamiento en las juntas, se observa que, en el segundo caso, a pesar de que el coeficiente de seguridad geométrico impuesto sea de 1, la trayectoria no es tangente al intradós y al extradós. ¿Por qué motivo? Porque no es posible encontrar una línea de empujes que se ajuste a dicho CSG sin provocar antes la rotura de los otros arcos o machones implicados.

Nota 2: Tampoco es posible encontrar un mecanismo de colapso. ¿Por qué? La estructura para un CSG de 1 es estable por sí misma hasta un valor de $\mathrm{Pi} / 9.95$ para el rozamiento (figura 9).

\section{Caso A2:}

Como en el cálculo manual se había adoptado la hipótesis de ceñir la trayectoria de cargas al núcleo central de inercia (suponiendo que las posibilidades de trabajo a tracción de la fábrica son despreciables), por tratarse de una solución más conservadora que trabajar con un análisis plástico puro o análisis límite en el que la resistencia a compresión de la fábrica se considera infinita, se retoma esta opción en Maple para comparar los resultados de la trayectoria de empujes con el cálculo manual. Por ello, bajo este criterio de CSG de 1 en soportes (resultante de acciones dentro del espesor del machón) y 3 en arcos, se intenta conocer cuál es el ángulo de rozamiento mínimo necesario para lograr la estabilidad del conjunto.

Se aprecia que, al aumentar las exigencias de seguridad geométrica del arco, aumentan también las exigencias del coeficiente de rozamiento en la junta. No obstante, resulta tranquilizador observar que tanto los CSG adoptados como los ángulos entran dentro de los estándares de este tipo de construcciones. Al igual que en el caso A1, se parte del límite inferior de $30^{\circ}$, es decir $\mathrm{Pi} / 6$, y se constata que para este valor la estructura es estable por sí misma sin necesidad de atirantado, es decir $F x=0$ (figura 10). Sin embargo, a partir de ese momento y si se continúa decreciendo el denominador hasta llegar al límite de Pi/6.6, el 
Estudio y aplicación de diferentes metodologías de cálculo para el conocimiento gradual del proceso constructivo y comportamiento mecánico de una arquería de la Mezquita de Córdoba María Escalada Marco-Gardoqui

problema sigue teniendo solución, pero Fx es distinto de 0 . Podría decirse que se genera un pequeño margen entre el valor de rozamiento para el que la arquería es autoestable y para aquel que demanda la presencia de una fuerza horizontal en la tercera piedra del machón de un valor de $5.41 \mathrm{KN}$.

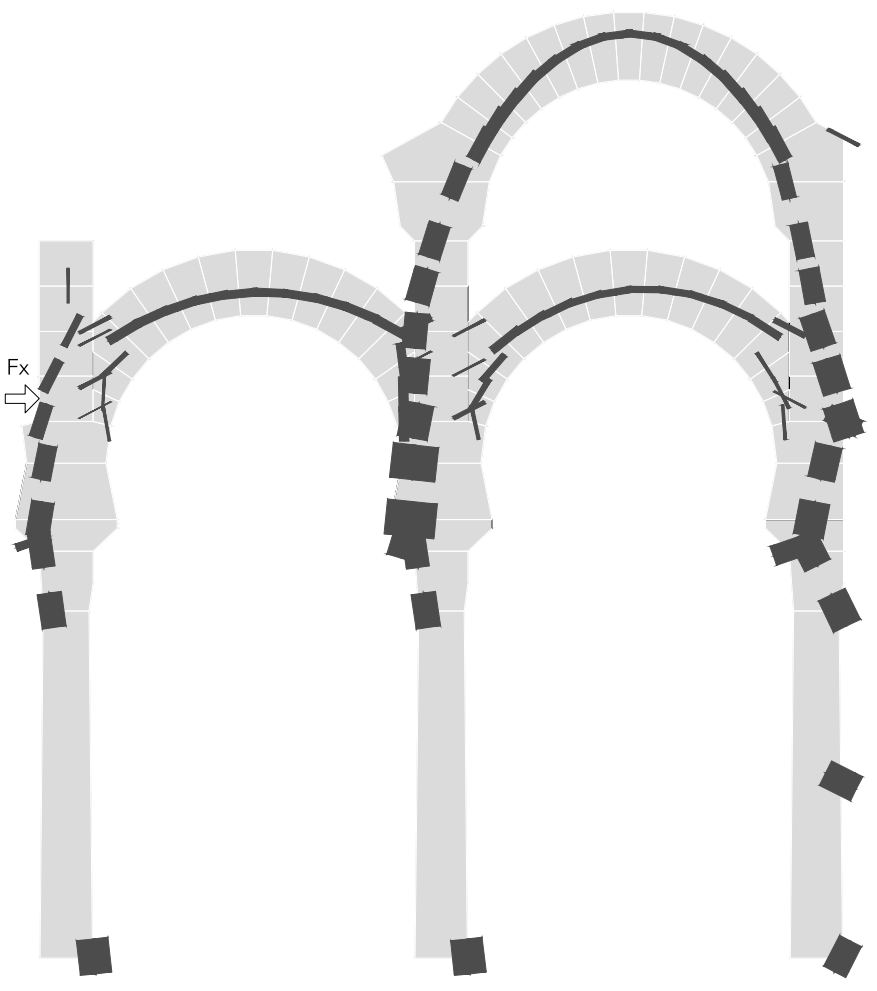

Fig. 10. CASO A2. Trayectoria de cargas para $C S G=3-a r c o s$, Ángulo de rozamiento: Pi/6.6 (Fx=5.4614)

No obstante, algunas razones para emplear el tirante sería:

1) El rozamiento que se puede esperar obtener en las juntas podría presentar una gran dispersión de valores dependiendo de la perfección de la talla de las piedras y ser ocasionalmente muy inferior al requerido. Tal y como se ha podido constatar en el taller de cantería, cualquier irregularidad debida a un lecho con cierta concavidad o convexidad que provoca que dos dovelas colindantes sean "tangentes" exclusivamente en unos puntos, afecta directamente al planteamiento.

2) Si se considera la posibilidad mencionada previamente de emplear el arco como apoyo total o parcial del andamiaje, necesariamente habrá que contar con algún coeficiente de seguridad adicional mayor que 1 para absorber las cargas generadas con este uso, con lo que tiene sentido emplear un contrarresto.

3) Aunque se prefiera personalmente el enfoque del análisis límite planteado en clase y en la bibliografía de referencia (Huertas y Heyman) que utiliza el coeficiente de seguridad geométrico de 1 , existen autores que recomiendan por varias razones mantener la trayectoria de cargas dentro del núcleo central de inercia (coeficiente de seguridad geométrico de 3 para arcos), como Valentín Quintas, tal y como lo expone en su publicación en Informes de la Construcción "Sobre los teoremas de rotura, sus corolarios y su aplicación al cálculo de arcos de fábrica".

Para valores inferiores a un ángulo de rozamiento de $30^{\circ}$ la estructura presenta mecanismos de rotura "clásicos" por tracción en la junta de los machones (figura 11) al salir la trayectoria de empujes de sus límites geométricos. Se puede apreciar que, a raíz de formarse la correspondiente rótula en el machón, el segmento superior de este se desplaza hacia el exterior y ocasiona que el arco del segundo nivel se abra formando el mecanismo de rotura típico para los de medio punto. El arco de herradura cuya construcción acaba de finalizar se ve empujado hacia la izquierda (por su derecha) y encuentra la resistencia opuesta por el puntal y tirante en el otro arranque, experimentando una rotura más cercana a la típica en arcos apuntados. 
Estudio y aplicación de diferentes metodologías de cálculo para el conocimiento gradual del proceso constructivo y comportamiento mecánico de una arquería de la Mezquita de Córdoba María Escalada Marco-Gardoqui

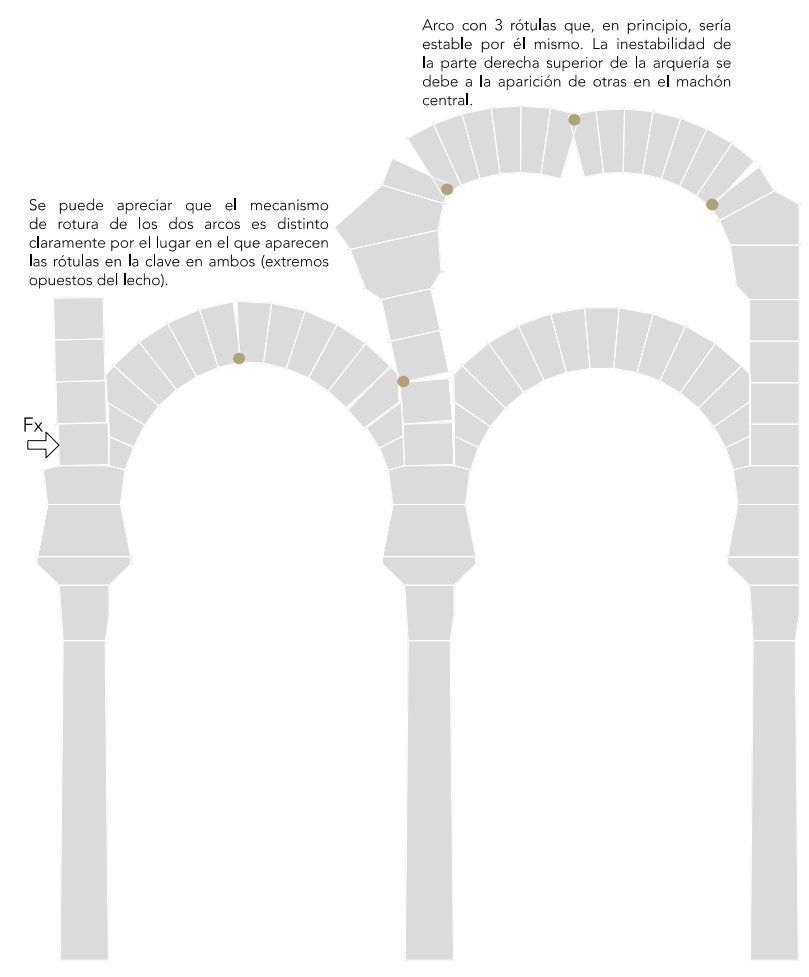

Fig. 10. CASO A2. Mecanismo de rotura para $C S G=3$-arcos, Ángulo de rozamiento: Pi/6.6 (Fx=0)

Con la trayectoria de cargas representada por el programa, aparentemente solo se formaría inicialmente una rótula en la junta mencionada, así que se hace la suposición de que a partir de una estimación de giro que pueda producirse en dicho punto e implementando condiciones de contacto en el resto de lechos es como el programa determina la sucesión de mecanismos de colapso mencionados.

\section{CASO ESTUDIO B:}

Con cimacios sin apear, calcular el empuje mínimo.

Bajo el criterio de coeficientes de seguridad geométricos de 1 en soportes y arcos, se intenta conocer cuál es coeficiente de rozamiento mínimo necesario para lograr la estabilidad del conjunto, llegándose a determinar que es $\mathrm{Pi} / 4.3$.

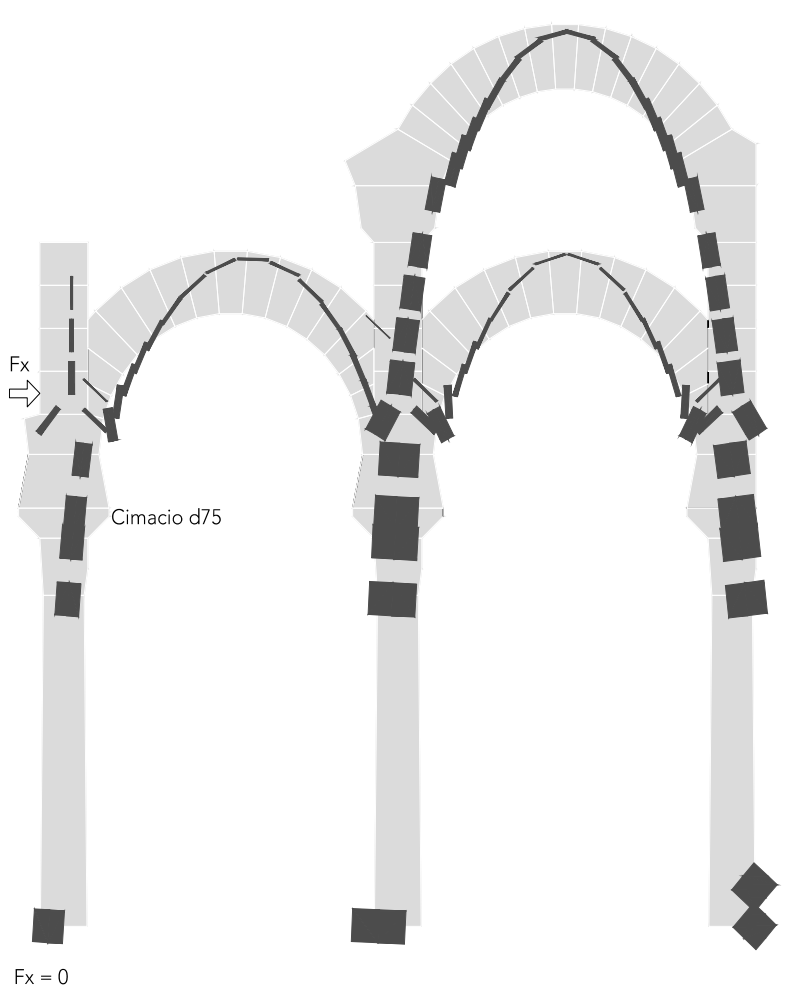

Fig. 11. $C A S O$ B. Trayectoria de empujes para $C S G=1$, Ángulo de rozamiento: Pi/4.3

Como se puede apreciar, se trata de un ángulo elevado de $41.8^{\circ}$, ligeramente por encima del límite superior del rango que se ha supuesto razonable al inicio de este apartado. Aunque quizás pueda alcanzarse con un tratamiento cuidadoso de los lechos en taller de cantería no parece particularmente prudente ni acorde con la filosofía de la construcción de la mezquita, donde primaban valores como la economía de medios y rapidez de construcción, centrando la atención en todo momento al detalle en los acabados visibles, no en la superficie interior de las juntas. Esto lleva a validar la hipótesis inicial adoptada de un modo intuitivo de que en algún momento de la construcción (como he planteado en el apartado 1) sería de recibo disponer de algún tipo de apuntalamiento provisional en los cimacios. Visto que el ángulo de rozamiento necesario resulta irreal de entrada, no merece la pena ajustar para esta hipótesis el dimensionado de un posible tirante. 
Estudio y aplicación de diferentes metodologías de cálculo para el conocimiento gradual del proceso constructivo y comportamiento mecánico de una arquería de la Mezquita de Córdoba María Escalada Marco-Gardoqui

\subsection{SAP2000}

Una limitación que tiene Maple es la dificultad para el cálculo de las tensiones en los lechos, para lo cual es necesario extraer los valores de axiles y cortantes y continuar por otras vías. Se propone un modelo de elementos finitos en SAP2000, donde los parámetros más relevantes a definir de los materiales son el módulo de elasticidad y la resistencia a compresión. Debido a los límites de extensión del trabajo y teniendo en cuenta que era la primera vez que se funcionaba con el programa, no se vio posible precisar mucho en el modelado, y se procedió a simplificarlo. En la imagen siguiente se puede apreciar el resultado del cálculo del primero modelado y todos los errores cometidos que se han ido identificando se describen a continuación.

Esto significa que en un primer momento no se han desarrollado micromodelos que recreen con detalle las diferentes propiedades de las juntas de mortero y de las piezas de fábrica y que el modelo de homogeneización de los materiales tampoco entra en un gran refinamiento. Se emplea la formulación linealelástica del método de los elementos finitos que lleva implementado SAP2000 por defecto sobre el conjunto de piezas de fábrica y juntas de mortero consideradas como único material homogéneo e isótropo. Sí se diferencia entre fábrica de ladrillo y piedra, y se intenta, en la medida de lo posible, acercarse al comportamiento de las juntas diseñando el mallado de los elementos finitos de tal manera que los nodos de estos coincidan alineados sobre ellas, discretizando cada dovela en 4 unidades.

Se analiza la estructura cuando está construido el arco de herradura del segundo vano (sometido únicamente a su propio peso) y los dos contiguos (sometidos a las cargas del muro y de la cubierta), empleando los límites inferiores en los módulos de elasticidad de los materiales (figura 12).

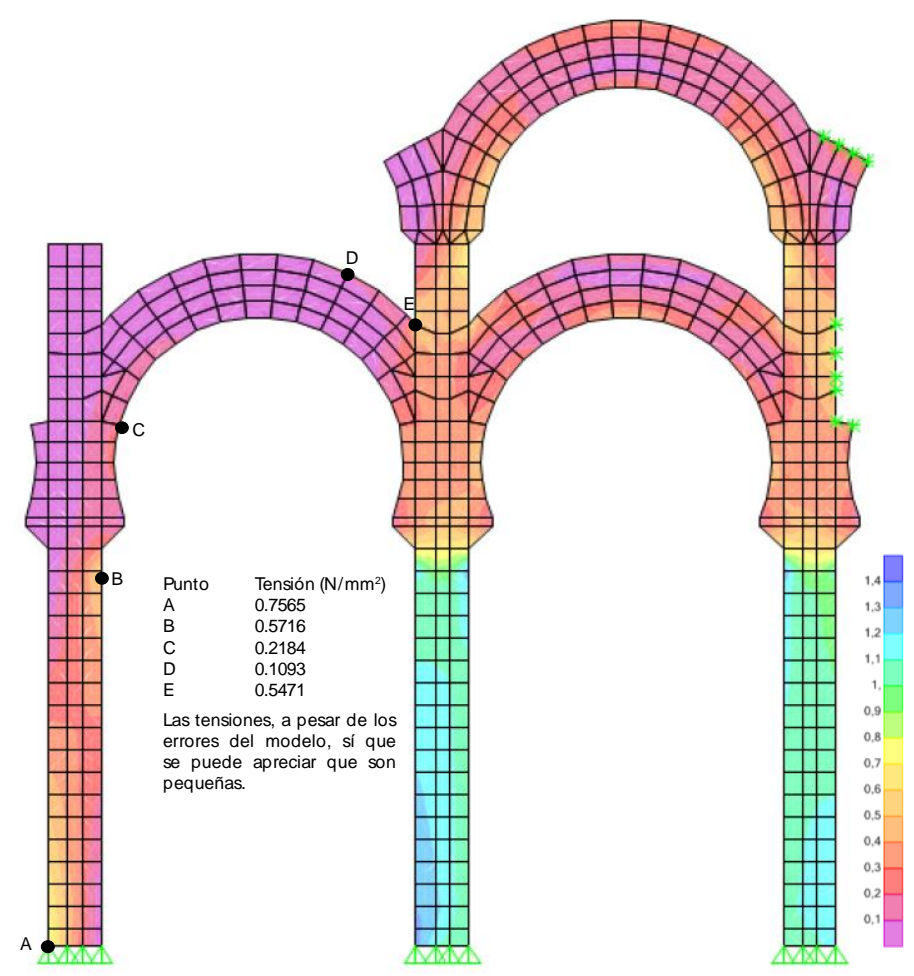

Fig. 12. Diagrama de tensiones primero-erróneo

Debido a la forma de trabajo del programa, si bien se pueden definir elementos tipo Frame o Barra con un comportamiento no lineal, es decir, entre otras propiedades, que trabajen solo a tracción o a compresión, este no permite proceder igual con elementos tipo Shell o Área, que son los que constituyen el modelo de fábrica de las arquerías de la Mezquita. Debido a esto, SAP2000 trabaja sobre hipótesis lineales y supone que los citados elementos funcionan tanto a tracción como a compresión, por lo que sobre todo en el tramo central del arco, aparecen trabajando de forma similar a una viga, con esfuerzos de flexión en el lecho (con una parte comprimida y otra traccionada). Aunque adoptando una hipótesis elastoplástica como se ha venido haciendo en 
apartados anteriores se suponer a la fábrica cierta resistencia a tracción y comprobar que esta no se supera en el modelo, al no encajar bien las hipótesis de partida con las suposiciones del análisis límite que sí se han venido usando para el cálculo manual (incluso cuando suponemos una distribución lineal de tensiones en los lechos y comprobamos dichas tensiones sobre hipótesis elástica con valores de resistencia tracción-compresión distintos de 0 - infinito la trayectoria de cargas la hemos trazado previamente suponiendo que los materiales trabajan predominantemente a compresión), el análisis que arroja el programa no resulta lo bastante representativo de la forma de trabajo real de la estructura.

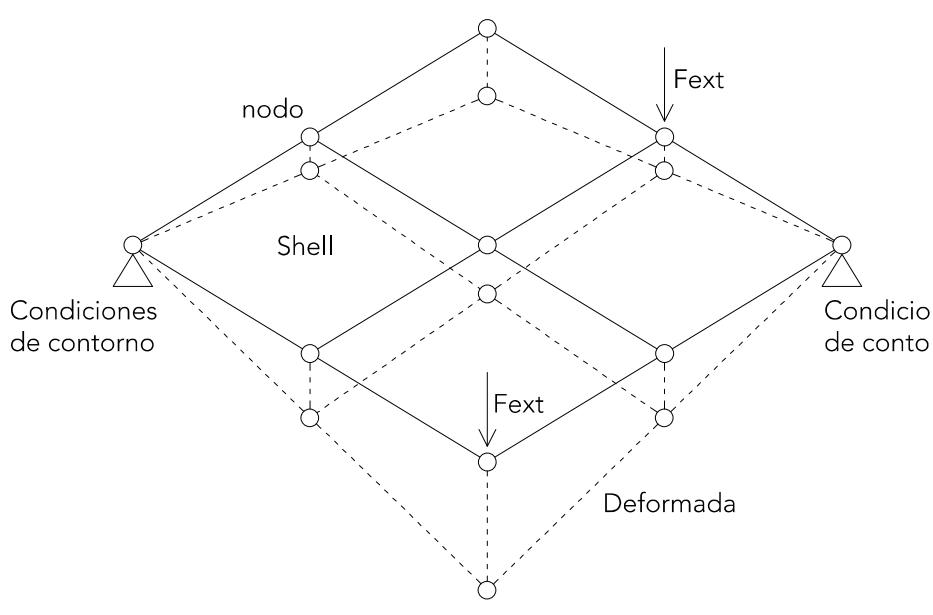

Fig. 13. Deformaciones de un elemento finito

Además, el programa no permite reflejar bien, tal como lo se ha modelado, los efectos de las juntas entre piezas, sencillamente supone todo como un continuo, impone la compatibilidad de deformaciones en los nodos que sirven de unión entre elementos y resuelve el problema en régimen elástico (figura 13). Hay que corregir el modelo y modelizar de alguna manera la junta para extraer conclusiones fiables [9].
A grandes rasgos, el método de elementos finitos relaciona las fuerzas aplicadas y para las condiciones de contorno, impone las condiciones de compatibilidad en los nodos intermedios del modelo, y lo que ocurre dentro de los elementos lo saca mediante funciones de interpolación.

\section{RESULTADOS}

\subsection{GEOGEBRA}

Se termina de trazar la línea de empujes hasta la cota de la cara superior del cimacio, y mediante el desplazamiento del punto focal se llega a la situación límite que se presenta en la imagen. Se puede determinar que, a priori, es posible contener dentro de la geometría del arco una trayectoria de mínimo empuje (con un coeficiente de seguridad igual o mayor que 3 para el arco), por lo que el arco sería autoestable y no necesitaría de elementos auxiliares de contrarresto durante el montaje. Sí que es verdad que la trayectoria de cargas llega bastante excéntrica al pilar, por lo que afirmar esto no resulta del todo del lado de la seguridad. Habría que afinar más en el cálculo y considerar el Geogebra como una herramienta orientativa, ya que cualquier mínimo deslizamiento de los puntos FOCO y DES (inicio de la trayectoria de empujes en el eje central de la clave del arco) tiene consecuencias gráficas sustanciales. Por ello, se continua con un estudio más detallado. 


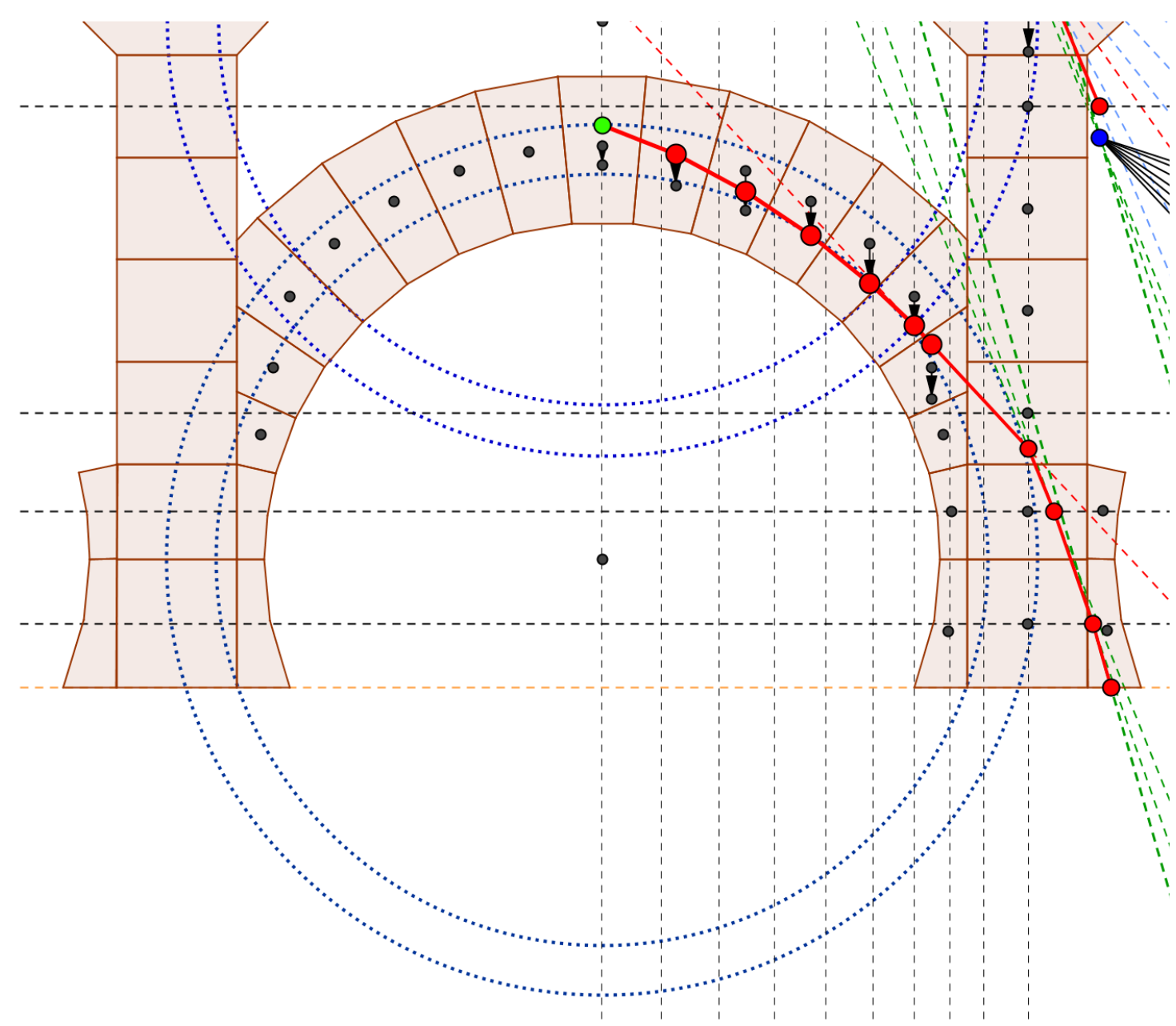

Fig. 14. Línea de empujes ajustada al núcleo central de inercia

\subsection{EXCEL}

Para el esquema de cargas aplicado se puede trazar una trayectoria de mínimo empuje $(\mathrm{Fh}=$ 4.427 KN) que quepa dentro de la geometría del machón, por lo que en principio, el arco de herradura es estable por sí mismo y no necesita de elementos auxiliares de contrarresto de empujes durante el montaje (la carga llega al cimacio bastante excéntrica como en la propuesta de Geogebra).

Sin embargo, también existe la posibilidad de considerar que una vez construido el arco, este pudiera servir para el apoyo total o parcial de plataformas de trabajo que facilitaran la construcción del superior de medio punto con el empleo del mínimo andamiaje posible. Una hipótesis puede apreciarse en el croquis adjunto. Aunque se es consciente de que diferirá enormemente de la solución adoptada en la época, se ha considerado positivo ejemplificar el concepto para la justificación de la solución adoptada. En un caso como este, en el que se incrementan las cargas actuantes en el sistema, podría ocurrir que la línea de empujes se saliera de la geometría del machón, como sucede en el dibujo de la máxima fuerza horizontal, $F h_{\text {máx }}=11.27 \mathrm{KN}$, por lo que se estima oportuno la colocación de un tirante en el punto donde la línea de

Advances in Building Education / Innovación Educativa en Edificación | ISSN: 2530-7940 | 
Estudio y aplicación de diferentes metodologías de cálculo para el conocimiento gradual del proceso constructivo y comportamiento mecánico de una arquería de la Mezquita de Córdoba María Escalada Marco-Gardoqui

empujes se sale fuera (o en un punto superior) para verticalizar la carga. Se considera también oportuno el apuntalamiento del pilar a la altura del cimacio (aprovechando los entrantes de las esquinas) para evitar inestabilidades durante la puesta en obra de las piezas que constituyen el machón y el arco de herradura (figura 15).

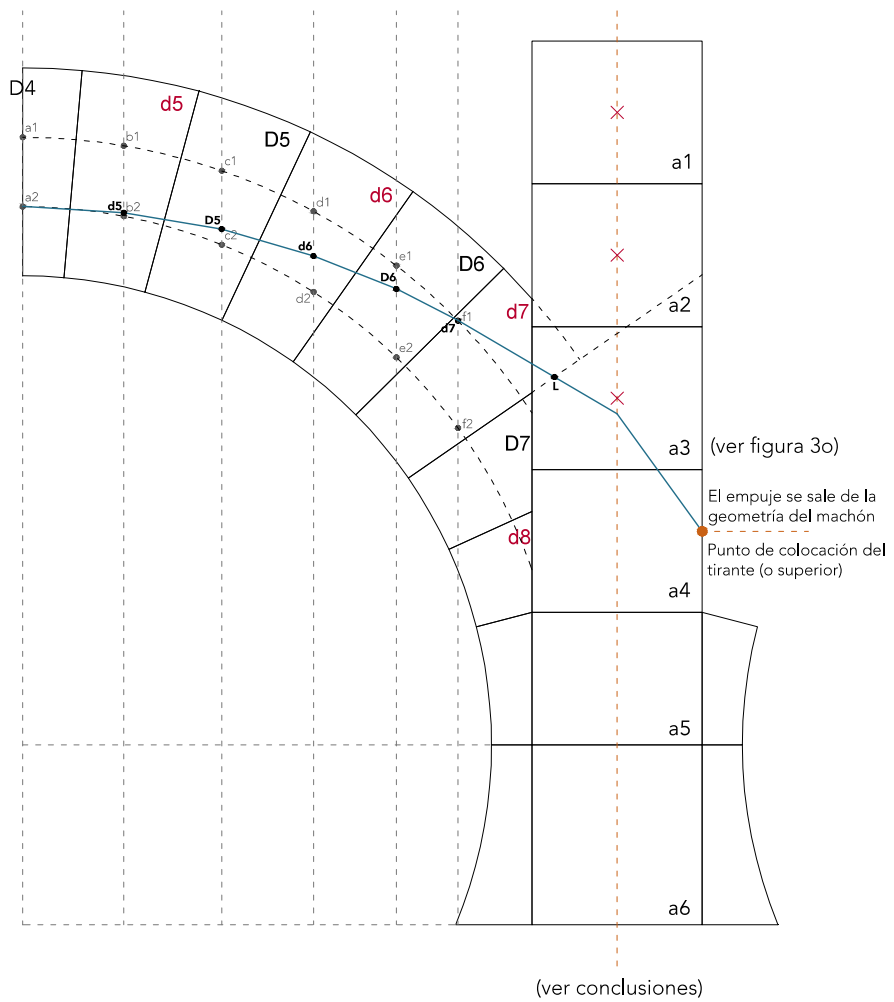

Fig. 15. Línea de empujes máxima

Es imposible determinar a ciencia cierta no solo qué tipo de tirantes o puntales se disponían en obra en la época, ya que una vez aventurada una hipótesis sobre el particular, tampoco es sencillo establecer sus dimensiones exactas. Esto es debido a que, aunque es posible calcular el coeficiente de seguridad para un conjunto de cargas dadas en el arco, pequeñas variaciones de geometría, materiales, etc. podrían alterar dicho valor de forma significativa, a pesar de que la construcción siguiera siendo estable. Así, solo se puede afirmar la necesidad de disponer o no tirantes cuando no se logra encajar la trayectoria de cargas a partir del arranque arco dentro de los machones.

Además, la cuantía del tirante puede variar significativamente según el coeficiente de seguridad con el que se quiere que este trabaje. En la actualidad, funcionando con valores entre 1.5 y 3 para los medios de ejecución auxiliares, estadísticamente se ha logrado reducir el número de accidentes laborales en obra a un margen que nuestra sociedad considera tolerable. Resulta difícil aventurar con qué margen les parecía razonable trabajar en el periodo de ejecución de la mezquita. Por tanto, lo que se hará en este ejercicio es ofrecer el rango, desde el valor mínimo indispensable para garantizar la estabilidad hasta aquel que suponga que se aprovechan al máximo las posibilidades de la estructura, empleando el mismo coeficiente de seguridad que el arco [10].

\section{Cálculo del tirante:}

\section{Caso 1}

Se predimensiona la estructura de contrarresto auxiliar empleando el coeficiente de seguridad 1 (que ya lleva implícito un coeficiente de seguridad 3 del arco por haber calculado la línea de empujes dentro del núcleo central de inercia) para una clase de madera C24, correspondiente al pino común por desconocimiento de aquella empleada en la época y una fuerza horizontal Fh de 9.172 $\mathrm{KN}$, de acuerdo con la trayectoria descrita en el apartado del mínimo empuje.

$$
\text { Área }=\mathrm{N} / \sigma
$$

$$
\text { Área }=9.172 \mathrm{KN} / 2.4 \mathrm{KN} / \mathrm{cm}^{2}=3.822 \mathrm{~cm} 2
$$

Sección cuadrada de $\sqrt{ } 3.822 \mathrm{~cm}^{2}=1.95 \mathrm{~cm}$ de lado

\section{Caso 2}

Se predimensiona la estructura de contrarresto auxiliar empleando el mismo coeficiente de 
Estudio y aplicación de diferentes metodologías de cálculo para el conocimiento gradual del proceso constructivo y comportamiento mecánico de una arquería de la Mezquita de Córdoba María Escalada Marco-Gardoqui

seguridad que el arco cuando solo está sometido a su peso propio, esto es:

$$
\begin{gathered}
\nu=e_{\text {real }} / \text { elimite }=0.5416 \mathrm{~m} / 0.1115 \mathrm{~m}=4.86 \\
\text { Área }=\mathrm{N} / \sigma \\
\text { Área }=\left(9.172 \mathrm{KN} \times \begin{array}{c}
4.86) / 2.4 \mathrm{KN} / \mathrm{cm}^{2}=18.57 \\
\mathrm{~cm} 2
\end{array}\right.
\end{gathered}
$$

Sección cuadrada de $\sqrt{ } 18.57 \mathrm{~cm}^{2}=4.31 \mathrm{~cm}$ de lado

Como se puede ver en ambos casos, las escuadrías de los tirantes son bastante reducidas, por lo que la cantidad de madera a emplear para lograr la estabilidad del arco con este medio auxiliar de contrarresto de empujes no supondría un gasto sustancial, más si diseñaron algún sistema de uniones que permitía la reutilización de los mismos.

\subsection{MAPLE}

\section{CASOS A1 y A2:}

Hay que tener en cuenta que fuerzas que colaboran en el equilibrio de forma importante como el posible esfuerzo que desarrolle el tirante, o los apeos, o el rozamiento entre las piezas, se consideran por lo general a lo largo de este trabajo como fuerzas pasivas, es decir, que solo actúan en la medida de lo necesario para restaurar el equilibrio cuando se producen los movimientos adecuados en las estructuras que pueden movilizarlas.

Respecto a esto en particular, se puede comentar una pequeña diferencia del planteamiento entre el análisis realizado en Maple y aquellos llevados a cabo previamente mediante la estática gráfica en Autocad y Excel. En Maple, se realiza el análisis límite de forma que se encuentre aquel esfuerzo máximo que los elementos auxiliares que se quieren dimensionar podrían llegar a ejercer ocasionando la rotura de la estructura, es decir, se optimiza con el algoritmo BBMI (método simplex) el empuje que podría provocar el tirante. Aunque como se ha mencionado, estos elementos desarrollan esfuerzos pasivos, aunque este planteamiento lleve a resultados perfectamente aplicables (para dimensionado de medios auxiliares) resulta algo contraintuitivo: el mecanismo de rotura que se produciría así y que se puede apreciar en la figura resulta muy poco habitual en arcos de herradura o de medio punto, siendo más común en arcos apuntados donde los apoyos están sobrecoaccionados y no existe peso suficiente sobre la clave. Sin embargo, es el que parece más probable al cambiar el comportamiento resistente con la inclusión de tirantes y/o apeos.

En el análisis previo, se lleva a cabo el análisis límite centrado en la estabilidad de la estructura y a partir del rango de empujes obtenido y las cargas necesarias para equilibrarlo se dimensionaban los elementos auxiliares, es decir, se optimizaba hacia máximos el empuje que puede provocar el arco para el sistema de cargas considerado.

Este enfoque puede resultar un poco más farragoso pero, a cambio, encaja mejor a priori con una idea intuitiva del problema [11]. Así, el mecanismo de rotura que se produce resulta el más habitual para arcos de medio punto o herradura cuando se forman rótulas permitiendo la caída de la clave a favor de la gravedad.

En cualquier caso, los dos enfoques llevan a similares resultados. El empuje mínimo obtenido con el cálculo de Autocad y Excel para el arco de herradura es de $4.427 \mathrm{KN}$ y en Maple, considerando el rozamiento y analizando la estructura en su globalidad, de $5.41 \mathrm{KN}$. Se podría concluir que no es una diferencia significativa y que esta puede venir derivada de la simetría ideal de cargas que se propuso en el ejercicio del apartado anterior. 


\begin{abstract}
Estudio y aplicación de diferentes metodologías de cálculo para el conocimiento gradual del proceso constructivo y comportamiento mecánico de una arquería de la Mezquita de Córdoba María Escalada Marco-Gardoqui
\end{abstract}

\section{CASO B:}

Para poder predimensionar el apeo resulta necesario conocer los esfuerzos en los cimacios para $\mathrm{CSG}=1$ para $\mathrm{Pi} / 9.95$ (figura 16).

Para obtener los valores numéricos de las fuerzas en los nodos y una gráfica de la pieza estudio, es necesario escribir lo siguiente en Maple:

$$
\begin{gathered}
n d:=75: \text { miModelo[2][nd]; } \\
\text { dibujoD(miModelo,dov=nd..nd); } \\
\text { dibujoEsf(miModelo,nd); S; (así se obtienen } \\
\text { los módulos) }
\end{gathered}
$$

Se predimensiona la estructura (cálculo a pandeo) empleando $C S G=1$ e intentando conseguir una sección cuadrada. La longitud estimada del apeo es de $3.5 \mathrm{~m}$, considerándose el caso más desfavorable de no contar con elementos de rigidización intermedios que acortaran la longitud de pandeo. Para el cálculo de la compresión, la resultante del empuje mínimo de $26.98 \mathrm{KN}$ se divide entre 2, por suponerse dos puntales a $45^{\circ}$ en planta.

$$
\begin{gathered}
\text { Lado: } 80 \\
\text { Inercia: } 3413333.3 \\
\text { Área: } 6400 \\
\text { Radio de giro: } 23.0940 \\
\text { Longitud: } 3500 \\
\text { Esbeltez. Mecánica: } 151.554 \\
\text { X: } 0.14 \\
\text { fc, } 0, \mathrm{k}: 22 \\
\text { Compresión: } 13490 \\
\text { oc,0,d: } 2.107 \\
\text { Comprobación: } 0.68 \\
\text { (medidas en mm y N) }
\end{gathered}
$$

Se descompone la fuerza $F=-29.472 \mathrm{KN}$ en los ejes de acuerdo con la inclinación que se quiere dar al apeo. Se "desprecia" la componente horizontal que serviría para dimensionar la resistencia cortante del apeo y se tiene en cuenta para el predimensionado únicamente el axil, de $26.98 \mathrm{KN}$.

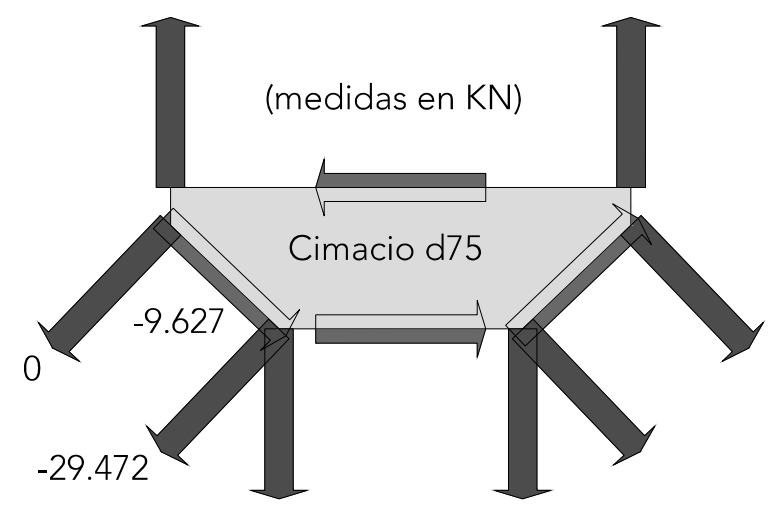

Fig. 16. CASO B1. Esfuerzos en cimacio izquierdo

\subsection{SAP2000}

Para conseguir que el modelo de cálculo por método de los elementos finitos refleje mejor la realidad, se puede proceder de varias maneras en SAP2000:

1. Definiendo los elementos con un comportamiento no lineal (por capas)

2. Definiendo las juntas entre elementos con un comportamiento no lineal (resistencia muy elevada a compresión y nula a tracción).

Debido a que resultaba más fácil de modelar y para intentar incorporar la corrección comentada en clase, se ha optado por la segunda. No obstante, debido a lo trabajoso de esta opción y a la falta de tiempo, se ha llevado a cabo solo en las dovelas del arco de medio punto. Se ha entendido este ejercicio como una introducción al trabajo de los elementos finitos que espero que en un futuro próximo me pueda servir para abordar problemas más complejos en el trabajo de fin de máster.

Así, las diferentes dovelas se modelan internamente con elementos tipo Shell lineales como los que se han venido utilizando, pero las juntas entre ellas se modelan mediante 
elementos tipo Gap (figura 17). Estos últimos son sencillamente del tipo "spring" (muelle) pero se comportan de forma no lineal porque solo trabajan a compresión. Aunque no tiene mayor relevancia para el trabajo, señalar que su nombre procede del hecho de que se puede definir un hueco entre los nodos que une el Gap y que este solo empieza a trabajar cuando la distancia entre los dos puntos es menor al hueco definido inicialmente.
Generalmente y por lo que he podido observar en internet sobre el tema, esta opción se utiliza para simular la interacción entre estructuras y el terreno o cierto tipo de elementos de amortiguación o relleno dispuestos en juntas entre edificios en caso de sismo.

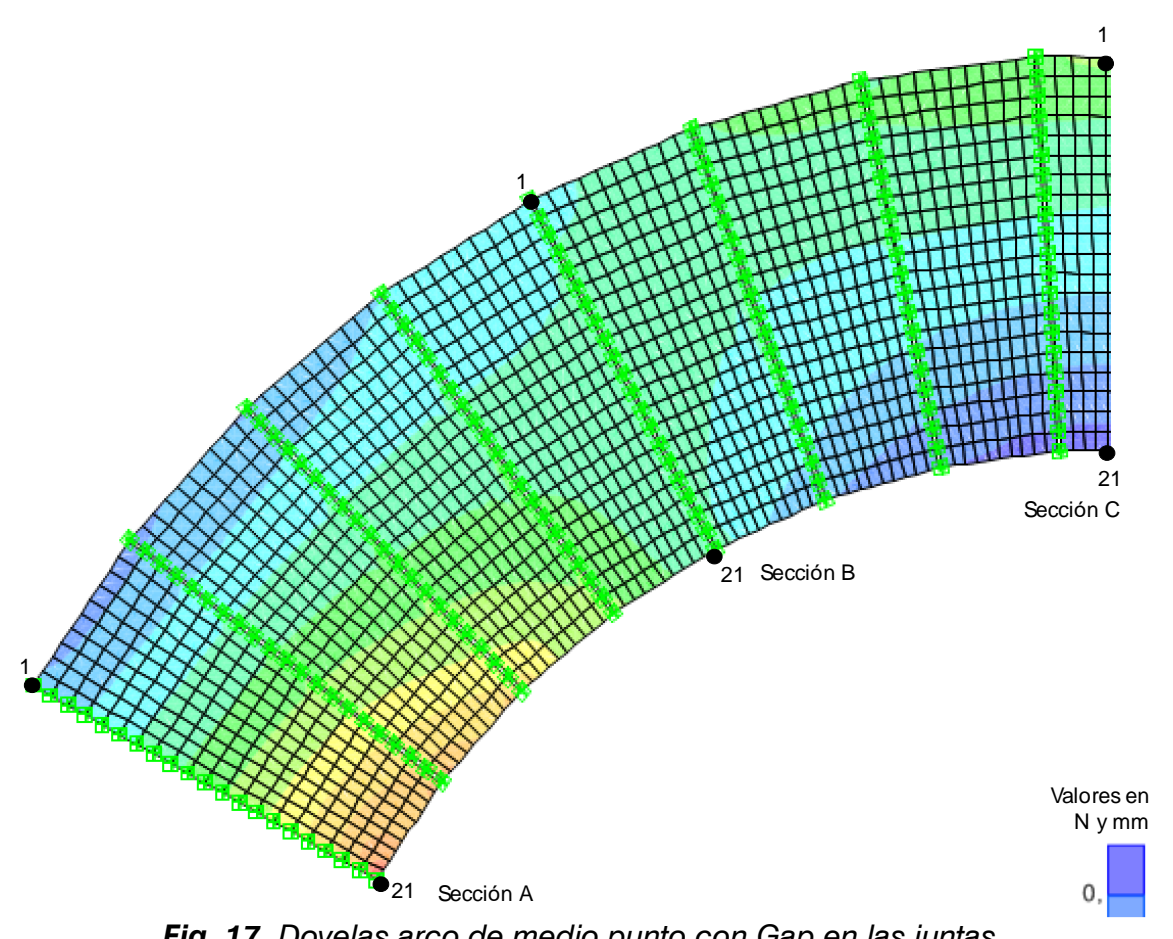

Fig. 17. Dovelas arco de medio punto con Gap en las juntas

El comportamiento del Gap en los demás aspectos es idéntico al de un muelle, siendo dada la relación entre la tensión aplicada y la deformación que experimenta el elemento según cada uno de sus grados de libertad en ejes locales por una constante de rigidez. LEY DE HOOKE: la relación entre la fuerza y deformación es lineal:

\section{Ut tensio sic vis $F=-k \cdot \delta$}

donde $\mathrm{k}$ es la constante de rigidez del muelle, $\delta$ la deformación y $\mathrm{F}$ la fuerza aplicada.
Cuando se define el comportamiento de los Gap estableciendo como fija su longitud en los tres grados de libertad correspondientes a su desplazamiento en los ejes locales (U1, U2, U3) y movimiento libre en los grados de libertad correspondientes a su rotación entorno a estos ejes, funcionan simplemente como un artificio para sacar, en la medida de lo posible, los esfuerzos de tracción del modelo. Aunque internamente los elementos tipo Shell siguen modelados de forma elástica y pueden presentar alguna tracción, al no poder transmitirse estas por las juntas, su valor es bajo y el comportamiento global del modelo

Advances in Building Education / Innovación Educativa en Edificación | ISSN: 2530-7940 |

http://polired.upm.es/index.php/abe

| Cod. 0021 | Septiembre - Diciembre 2017 | Vol. 1. № 3 | pp. 80/106 | 
se corresponde en gran medida con lo esperado.

Otras mejoras que no se han mencionado pero que sí que se han incorporado al modelo son por ejemplo, la orientación de los ejes locales (figura 18), buscando un paralelismo a las directrices del perímetro de cada dovela para facilitar la lectura de la U1 y U2. Además, cada una de las dovelas se ha dividido en más elementos finitos (en el primero eran exclusivamente 4) para una mayor precisión de los resultados. (El análisis se ha hecho en 2D)

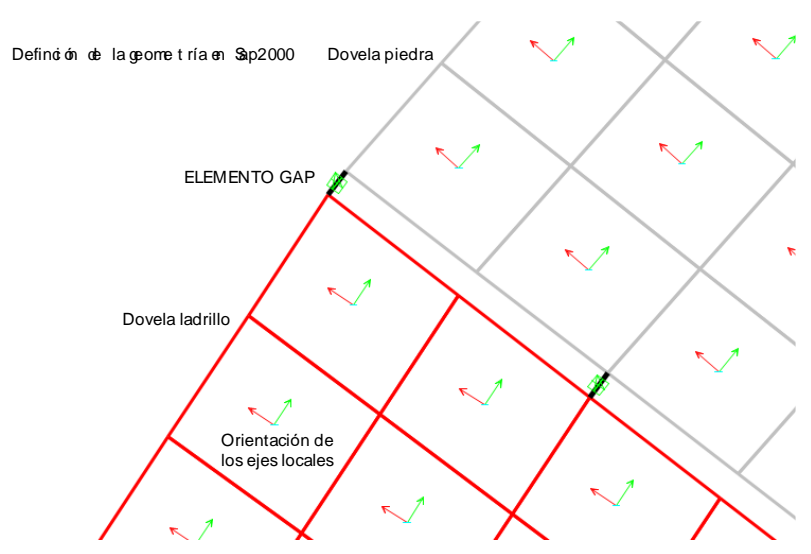

Fig. 18. Orientación de los ejes locales y gaps

Se podría afinar quizás más con las características de los Gap hasta representar de una manera muy realista las propiedades de la junta de mortero y comprobar si el modelo va bien con los valores de rozamiento que exigía Maple. Sin embargo, se decide continuar en este apartado con los datos obtenidos de los diagramas de tensiones anteriores.

Se escoge un punto próximo al apoyo del arco (figura 19). Se dibujan las posibles líneas de resistencia intrínseca L.R.I., que representan lo que aguanta el material según los parámetros de cohesión y ángulo de rozamiento interno. Al igual que pasaba con el módulo de elasticidad E (para el Sap2000), el ángulo de rozamiento entre piezas (para el Maple), determinar estos parámetros a ciencia cierta es muy difícil, así que habría que acotar razonablemente un rango de valores.

En verde: Un material sin cohesión y con ángulo de rozamiento interno de $53^{\circ}$ resiste este estado de tensiones.

En azul: Un material con cohesión $=8.79$ $\mathrm{KN} / \mathrm{m}^{2}$ aprox. $=0.009 \mathrm{MPa}$ y con ángulo de rozamiento interno de $40^{\circ}$ resiste este estado de tensiones.

En rojo: Un material con cohesión $=14.3$ $\mathrm{KN} / \mathrm{m}^{2}$ aprox. $=0.014 \mathrm{MPa}$ y con ángulo de rozamiento interno de $30^{\circ}$ resiste este estado de tensiones.

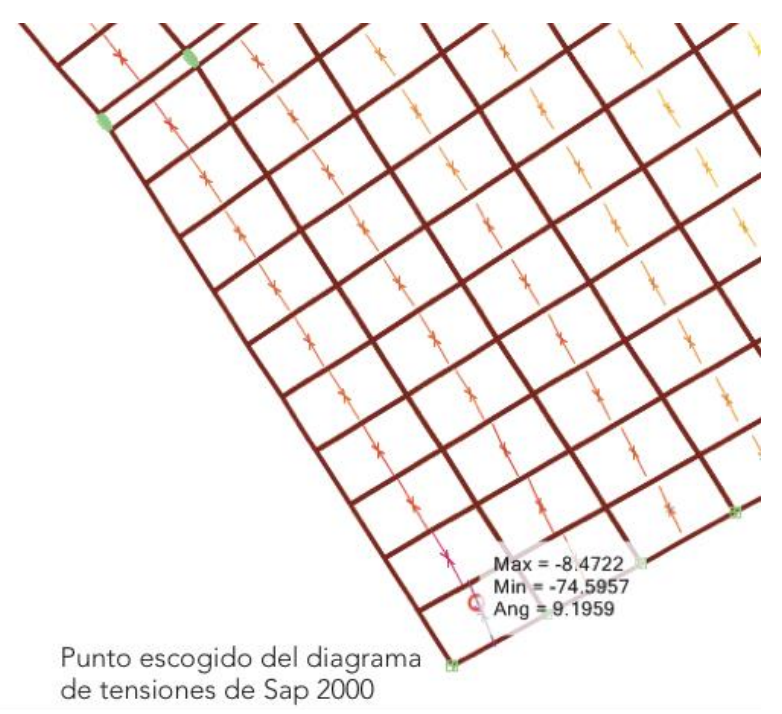

Fig. 19. Punto escogido para el dibujo de tensiones

Este círculo incluye varias combinaciones de tensión equivalentes entre sí, a saber (figuras 20 y 21 ):

- En tensiones principales (las dos normales), las citadas $S_{\text {máx }}=-8.43$ y $S_{\text {mín }}=-74.59$

- Una tensión normal de -41.32 y una tangencial de 33.2

- Una tensión normal de -22.88 y una tangencial de 27.3 , etc.

Choca un poco que sean equivalentes estados de tensión como los aquí citados, en los que los valores de tensión de un estado son 
menores que los del otro. Pero es que, a menos tensión normal, menos resistencia a tensión tangencial.

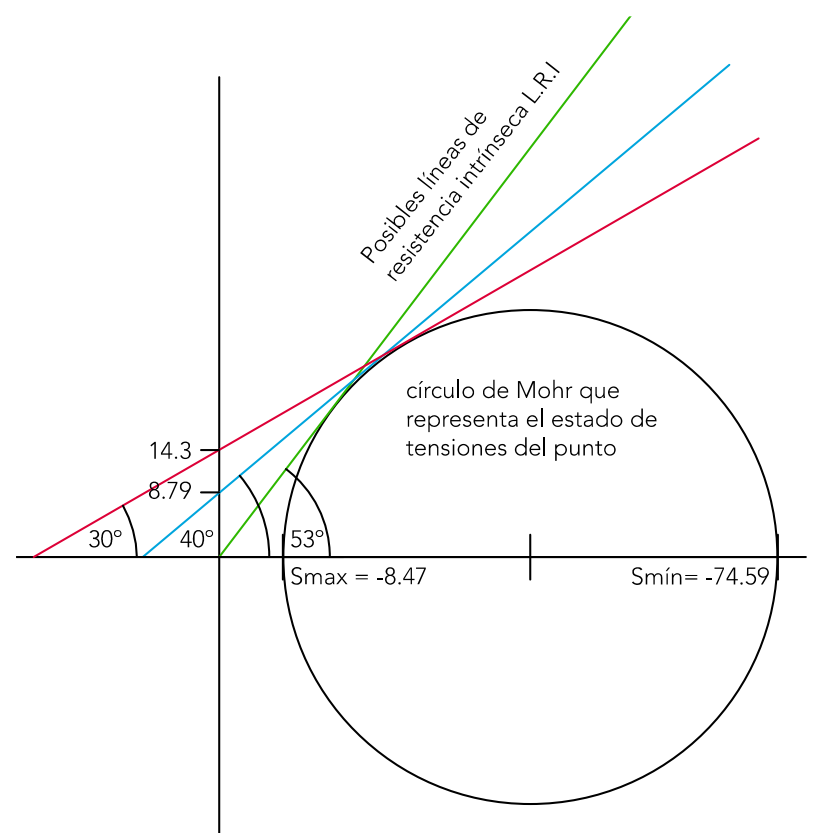

Fig. 20. Círculo de Mohr y líneas L.R.I

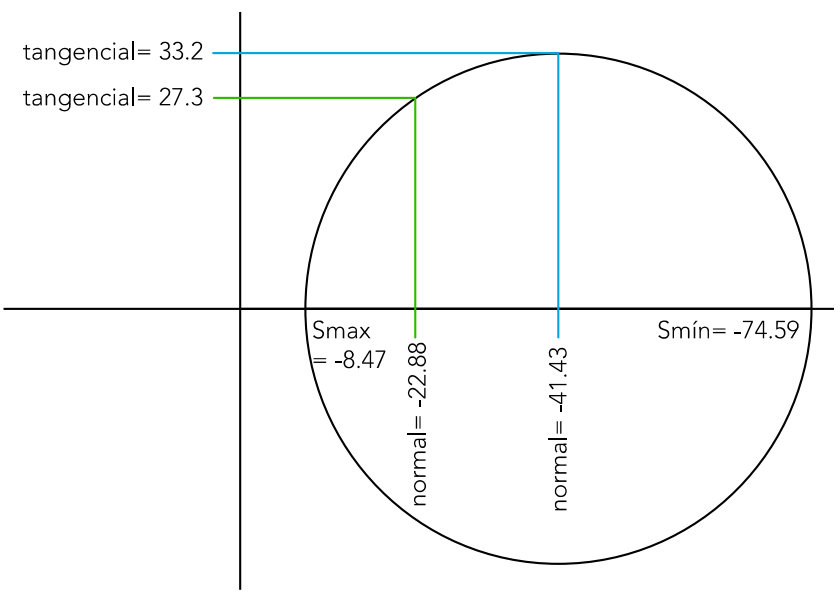

Fig. 21. Círculo de Mohr - tensiones

\section{CONCLUSIONES}

El proyecto docente planteado, basado en una organización del conocimiento estructural progresivo sobre un mismo ejercicio y en un entendimiento de la realidad constructiva del edificio (arquerías de la mezquita de Córdoba), supone un cambio en la manera de abordar la enseñanza de estas materias a escala universitaria, donde a menudo no existe esa transversalidad de disciplinas y se obvian conceptos imprescindibles de la concepción arquitectónica de los antiguos que dificultan la posterior comprensión de los resultados. Esto es, tiene implicaciones que trascienden el mero ámbito del cálculo numérico.

Aunque el proyecto contempla el empleo de software específico para el análisis de estructuras, también se busca capacitar al alumno para crear su propio modelo de optimización matemática, mucho más sencilla, a partir de hojas de cálculo Excel, favoreciendo así el trabajo autónomo, y promoviendo la búsqueda de planteamientos diferentes $y$ ampliables para la resolución del mismo problema y extrapolables a otros casos.

\section{Agradecimientos}

A José Ignacio Hernando, Fernando Magdalena y Joaquín Antuña, profesores de las asignaturas de Estabilidad y Métodos de Análisis I y II del master MUCTEH, por haber ayudado en la elaboración del modelo de Maple. 


\section{REFERENCIAS}

[1] Carl E. Wieman, "Large-scale comparison of science teaching methods sends clear message", PNAS -Proceedings of the National Academy of Sciences of the United States of America, vol.111 no 23, pp. 8319 - 8320, 2014

[2] G. Torregosa Gironés, "El desarrollo del sentido geométrico como una relación entre la visualización y el razonamiento configural", vol.70, pp.16-20, 2015

[3] L. Rubio, J. Prieto, J. Ortiz, "La matemática en la simulación con Geogebra. Una experiencia con el movimiento en caída libre", Revista Internacional de Investigación e Innovación Educativa, no 5, pp.93 - 94, 2016

[4] D. Mencías Carrizosa, "Verificación de la estabilidad de estructuras de fábrica mediante Geometría Dinámica", Sociedad de la Información no 47, pp. 1-7, 2014

[5] N. Tripathi, N. Srivastava, "Optimization problems solved by different platforms say optimum tool box (Matlab) and Excel Solver", International Research Journal of Engineering and Technology (IRJET), Volume: 04 Issue: 09, pp. $1284-1287,2017$

[6] S.Huerta, "Mecánica de las estructuras de fábrica: el enfoque del equilibrio", Informes de la Construcción, Vol. 56, no 496, pp. 74-88, 2005

[7] F. Magdalena Layos, "El problema del rozamiento en el análisis de estructuras de fábrica mediante modelos de sólidos rígidos", PhD Tesis. Dir. Huerta Fernández, Santiago y Hernando García, José Ignacio, 2013

[8] S.Huerta, "Arcos, bóvedas, cúpulas. Geometría en el cálculo tradicional de estructuras de fábrica", pp. 14-17, 75-77, 2004

[9] A.J. Mas-Guindal, "Mecánica de las estructuras antiguas o cuando las estructuras no se calculaban", pp. 73-80, 2011.
[10] J. Heyman, "El esqueleto de piedra", pp. 17-31, 1997.

[11] J. Antuña, "Creación de herramientas interactivas para la enseñanza de estructuras de edificación: Modelos virtuales de bóvedas nervadas" Proyecto de innovación educativa IE1415- 03015, financiado por la Universidad Politécnica de Madrid, 2014-2015

\footnotetext{
Advances in Building Education / Innovación Educativa en Edificación | ISSN: 2530-7940 | http://polired.upm.es/index.php/abe 